\title{
Detailed comparison of two popular variant calling packages for exome and targeted exon studies
}

The Genome Analysis Toolkit (GATK) is commonly used for variant calling of single nucleotide polymorphisms (SNPs) and small insertions and deletions (indels) from short-read sequencing data aligned against a reference genome. There have been a number of variant calling comparisons against GATK, but an equally comprehensive comparison for VarScan not yet been performed. More specifically, we compare 1) the effects of different preprocessing steps prior to variant calling with both GATK and VarScan, 2) VarScan variants called with increasingly conservative parameters, and 3) filtered and unfiltered GATK variant calls (for both the UnifiedGenotyper and the HaplotypeCaller). Variant calling was performed on three datasets ( 1 targeted exon dataset and 2 exome datasets), each with approximately a dozen subjects. In most cases, pre-processing steps (e.g., indel realignment and quality score base recalibration using GATK) had only a modest impact on the variant calls, but the importance of the pre-processing steps varied between datasets and variant callers. Based upon concordance statistics presented in this study, we recommend GATK users focus on "high-quality" GATK variants by filtering out variants flagged as low-quality. We also found that running VarScan with a conservative set of parameters (referred to as "VarScan-Cons") resulted in a reproducible list of variants, with high concordance ( $>97 \%)$ to high-quality variants called by the GATK UnifiedGenotyper and HaplotypeCaller. These conservative parameters result in decreased sensitivity, but the VarScan-Cons variant list could still recover $84-88 \%$ of the high-quality GATK SNPs in the exome datasets. This study also provides limited evidence that VarScan-Cons has a decreased false positive rate among novel variants (relative to high-quality GATK SNPs) and that the GATK HaplotypeCaller has an increased false positive rate for indels (relative to VarScan-Cons and high-quality GATK 
UnifiedGenotyper indels). More broadly, we believe the metrics used for comparison in this study can be useful in assessing the quality of variant calls in the context of a specific experimental design. As an example, a limited number of variant calling comparisons are also performed on two additional variant callers. 
2

3

4

\title{
Detailed Comparison of Two Popular Variant Calling Packages for Exome and Targeted Exon Studies
}

\author{
Charles D Warden ${ }^{1 *}$, Aaron W Adamson², Susan L Neuhausen², and Xiwei Wu ${ }^{3^{*}}$
}

5 'Department of Computational Medicine and Bioinformatics, University of Michigan, Ann Arbor, Ml 48109,

$6 \quad{ }^{2}$ Department of Population Sciences, ${ }^{3}$ Integrative Genomics Core, Department of Molecular and Cellular

7 Biology, City of Hope National Medical Center, Duarte, CA 91010

8 * To whom correspondence should be addressed. Tel: 626-256-4673; Fax: 626-471-3708; Email: cwarden@coh.org or 9 xwu@coh.org 
INTRODUCTION

11 Multiple studies have previously compared variant callers for short-read sequencing data (Bauer 12 2011; Cheng et al. 2014; Liu et al. 2013; O'Rawe et al. 2013; Pabinger et al. 2014; Pirooznia et al. 2014;

13 Yi et al. 2014; Yu \& Sun 2013). Many studies have indicated that the variant callers available in the

14 Genome Analysis ToolKit (GATK, DePristo et al. 2011; McKenna et al. 2010) show the best performance

15 (Bauer 2011; Liu et al. 2013; Pirooznia et al. 2014; Yi et al. 2014). This is in accordance with the popular

16 use of GATK for variant calling, especially for Illumina sequencing data (Boland et al. 2013; Li et al. 2014;

17 Linderman et al. 2014; Worthey 2013). However, there are reports showing other variant callers that can

18 outperform GATK, questioning the notion that GATK should be considered a gold-standard for variant

19 calling. For example, one study found that CASAVA outperformed the GATK UnifiedGenotyper when

20 calling single-nucleotide variants (Cheng et al. 2014) and another study showed that a novel algorithm

21 called Scalpel outperformed GATK HaplotypeCaller for indels (Narzisi et al. 2014). The use of multiple-

22 variant callers has also been proposed (Lam et al. 2012; O'Rawe et al. 2013; Pabinger et al. 2014; Yu \&

23 Sun 2013), but this will increase the run-time (or at least computational resources) necessary for analysis

24 (which can be especially important for large patient cohorts).

While the somatic variant calling function in VarScan (Koboldt et al. 2009; Koboldt et al. 2012) has been compared against other somatic variant callers (Roberts et al. 2013; Wang et al. 2013; Xu et al.

27 2014), most comparisons for single-sample variant calling (often used for identifying germline mutations)

28 did not directly compare GATK variant callers against VarScan. One study indicated that VarScan was

29 less accurate than the other variant callers (Cheng et al. 2014). Another study showed VarScan as being

30 more similar to the other variant callers but still ranked GATK as the best option (Yi et al. 2014). However,

31 VarScan has been used for variant calling in a large number of studies (Worthey 2013), and we

32 hypothesize that the simple, intuitive parameters can be helpful in establishing an optimal set of variants

33 for a given dataset. Also, as emphasized in this study, the run-time for VarScan should typically be shorter

34 than GATK. Therefore, we wished to determine if 1) the previous VarScan benchmarks can be

35 reproduced in our own analysis and 2) if use of non-standard parameters can improve the quality of the

36 VarScan variant calls. 
This study also examines the relative impact of pre-processing steps in GATK (specifically, the

38 indel realignment and quality score base recalibration steps). Therefore, we compared variant calls with

39 GATK and VarScan for each step separately, with both pre-processing steps, as well as without either pre-

40 processing step. There has been at least one previous study to showing that filtering can improve the

41 quality of variant calls (Carson et al. 2014), beyond the GATK quality score base recalibration. We

42 assessed whether running VarScan with different sets of parameters (using three different parameters

43 settings: see Methods) can also increase the accuracy of the resulting variant calls. Additionally, we have

44 used a simple filtering strategy for GATK variants (looking at all variants called versus filtering out variants

45 with a low quality flag), so there are two sets of variant lists for both GATK UnifiedGenotyper and GATK

46 HaplotypeCaller.

In order to avoid bias that could come from studying only a limited number of samples, variant calls were performed on 14 targeted exon (for 1000 genes) samples from the 1000 Genomes project (The 1000 Genomes Project Consortium 2012), 12 exome samples from the 1000 Genomes project (The 1000

50 Genomes Project Consortium 2012), and 15 Illumina exome samples from SRP019719 (O'Rawe et al.

51 2013). We believe this helps yield robust results both in terms of the number of samples studied per

52 cohort as well as variations in study design (i.e. the method of targeted sequencing). The 1000 Genomes

53 study was specifically chosen in order to test recovery of validated variants as well as to compare

54 concordance between samples subject to both targeted exon and exome sequencing.

In short, this study presents a detailed characterization between GATK and VarScan on 41 samples (with varying target designs), where each sample has 28 variant lists for comparison. Variant

57 lists are compared based upon the number of variants called, the proportion of novel variants (defined in

58 those absent from the 1000 Genomes Project (The 1000 Genomes Project Consortium 2012), Exome

59 Sequencing Project (Fu et al. 2013), and dbSNP (Sherry et al. 2001)) in the variant list, and the

60 reproducibility of variant calls using different technologies. A limited number of additional comparisons are

61 also performed in order to help illustrate how these metrics can be used to select the optimal variant caller

62 for a given dataset. This analysis demonstrates that a conservative set of parameters (referred to as

63 "VarScan-Cons") can be used to produce a reproducible list of variants from VarScan, and there is limited

64 evidence that VarScan-Cons has a lower false discovery rate among novel variants. This study also 
65 presents evidence that the GATK HaplotypeCaller may have a higher false discovery rate in calling indels

66 compared to the GATK UnifiedGenotyper.

\section{MATERIAL AND METHODS}

68 Sample Selection

69

70 al. 2011). Illumina exome samples were downloaded from SRP019719 (O'Rawe et al. 2013). 1000

71 Genomes Project (The 1000 Genomes Project Consortium 2012) data, abbreviated as $1 \mathrm{KG}$ in this 72 manuscript, was selected on the basis of having 1) exome data, 2) targeted exome data, 3) Omni SNP

73 chip data, and 4) validated SNPs. Among samples meeting those criteria, 12 samples were selected based upon 1) their presence in disparate populations (CEU: Northern and Western European Ancestry, CHB: Han Chinese, JPT: Japanese, and YRI: Yoruba/African) and 2) maximum number of validated SNPs

76 within each of the four selected populations.

1000 Genomes validated SNPs and Omni SNP chip .idat files were downloaded from the 1000

Genomes FTP site (ftp://ftp.1000genomes.ebi.ac.uk/vol1/ftp/). Likewise, target design files (for targeted exon and exome samples) were downloaded from this site. More specifically, the phase 3 design files were used to calculate coverage statistics for the exome samples, and validated variants come from ALL.chr20.exome_consensus_validation_454.20120118.snp.exome.sites.vcf.gz (pooled 454 PCR sequencing data). At the time this dataset was downloaded, the hg19 reference location for validated

83 SNPs was off-set by one (similar to indels in .vcf files), and this was taken into consideration during analysis. Targeted gene co-ordinates (for hg18) from P3_consensus_exonic_targets.bed were converted to hg19 coordinates using the LiftOver function in Galaxy (Blankenberg et al. 2001; Giardine et al. 2005;

86 Goecks et al. 2010).

Data Processing

Reads were aligned to a karotype-sorted hg19 reference (necessary for running GATK (McKenna

89 et al. 2010)) using BWA (v0.7.5a) (Li \& Durbin 2009). Prior to variant calling, singletons were filtered out

90 using samtools (v0.1.19) (Li et al. 2009), .bam files were coordinate sorted via Picard (v1.105,

91 http://picard.sourceforge.net//), and duplicates were removed via Picard. Prior to running GATK, read 
92 groups were added via Picard, and .bam file was re-ordered according to chromosome karyotype. Prior to

93 running VarScan (Koboldt et al. 2012), a pileup file was created via mpileup in samtools (Li et al. 2009)

94 and positions without any aligned reads were filtered out. These represent the minimum pre-processing

95 sets and are labelled as "No Preprocessing". Alignment statistics for these samples are shown in

96 supplementary tables (Table S1 for 1KG Targeted Exon, Table S2 for 1KG Exome, and Table S3 for

97 SRP019719).

98

There are three additional pre-processing pipelines that were considered for VarScan and GATK comparisons. "Realign Only" runs an indel realignment using GATK (using the RealignerTargetCreater and IndeIRealigner functions). "Recalibrate Only" uses GATK to recalibrate quality scores (using the

101 BaseRecalibrator and PrintReads functions). "Full Pipeline" runs the indel realignment functions and then 102 performs base recalibration.

MiSeq amplicon data (for individual K8108-49685s in the SRP019719 dataset) was processed in a similar pipeline as the array-based targeted sequencing data except that reads were first trimmed to 150 bp and PCR duplicates were not removed (since all reads were PCR duplicates). Also, unlike the SRP019719 exome data, GATK did not detect miscoded quality scores in the SRP019719 amplicon data

107 (so, the variant calling steps match the 1000 Genomes sample commands in Supplemental Text S1).

Human610 SNP chip data for individual K8101-49685s (paired with SRP019719 Illumina exome sample SRX265476) was reported in the Illumina "TOP" format, so the reverse complement of the allele was used when the IImnStrand and RefStrand did not match (as defined in the human610-quadv1_h.csv manifest file). Allele sequences were provided without respect to a reference sequence, so SeattleSeq

112 Variant Annotation (http://snp.gs.washington.edu/SeattleSeqAnnotation138/) was used to determine the

113 reference sequence to focus on variants that differ from the reference sequence (to make results

114 comparable to the Illumina sequencing variant calls). This is the latest version of the manifest file, but it

115 was designed using dbSNP 131 and some discordant SNPs were due to annotations where the forward

116 strand may vary from the hg19 reference sequence. However, this only affected a relatively small minority 117 of SNPs ( $<5 \%$ of variants).

118 Raw .idat files for 1000 Genomes Omni SNP chip samples were processed in Illumina® Genome 119 Studio $^{\text {TM }}$ (V2011.1). A pre-defined clustering file (HumanOmni2.5-4v1-Multi_H.egt) was used to call 
120 genotypes. Variants were exported in the "Plus" format (so, no genotype conversion as necessary).

121 Samples were annotated using the HumanOmni2.5-4v1-Multi_B.bpm manifest file, including the genomic 122 position in hg18 coordinates. Coordinates were converted to hg19 via liftOver in Galaxy (Blankenberg et

123 al. 2001; Giardine et al. 2005; Goecks et al. 2010) and reference sequences were determined for all on-

124 target probes $(n=2257)$ via SeattleSeq Variant Annotation

125 (http://snp.gs.washington.edu/SeattleSeqAnnotation138/). Each sample had 2179-2188 genotyped SNPs 126 recognized by SeattleSeq, with 477-616 non-reference alleles per sample.

\section{Concordance Definitions}

Concordance was defined as recovery of a set of lower-throughput variants (validated, SNP chip,

129 targeted exon, amplicon), except for the targeted exon technical replicates where concordance was

130 defined as (2* Number of Overlapping Variants) / (Sum of Variants from both samples). For clearity, we

131 therefore refer to recovery of lower-throughput results as the "recovery rate" in the results and reserve the

132 term "concordance rate" for only the technical replicate analysis. When defining variant concordance

133 between the SNP chip data and exome data, recovery of the known variant is counted as a concordant

134 variant (even if multiple variants are called at a given position).

135 Unlike most comparisons in this study, the SNP chip comparisons do not specifically focus on the

136 coding variants. As such, extraction of SNP chip variants within the target regions currently includes some

137 non-coding variants (such as intronic variants) that would falsely be called discordant if focusing only on

138 coding variants. SNP chip variants have been filtered to only include variants that vary from the reference 139 sequence.

140 Calling Variants

141 Variants were called using GATK (v.2.8.1) based upon established best practices (DePristo et al.

142 2011; Van der Auwera et al. 2002). Variants were called using both the UnifiedGenotyper (UG) and the

143 HaplotypeCaller (HC). For variant characterization, the set of all variants was considered (labelled as UG-

144 all in figures for the UnifiedGenotyper and $\mathrm{HC}$-all for the HaplotypeCaller) as well as only the high-quality

145 variants that didn't contain the "LowQual" flag in the .vcf file (labelled as UG-HQ in figures for the

146 UnifiedGenotyper and HC-HQ for figures for the HaplotypeCaller). In addition to the parameters described 


\section{PeerJ Reviewing Manuscript}

147 in the GATK best practices, variant calling for SRP019719 also required some additional parameters due

148 to the quality scores for the ENA reads (these extra parameters were not necessary for calling variants

149 from 1000 Genomes Project data). Parameters for calling 1000 Genomes and SRP019719 variants are 150 provided in Supplemental Text S1 and S2, respectively.

151 VarScan (v.2.2.8) variants were called using pileup2snp and pileup2indel. Three different sets of 152 parameters were used for calling variants. "VarScan: Default" specifies no additional parameters beyond

153 the minimal requirements. "VarScan: P-value" sets a p-value threshold of 0.05 , but specifies no additional 154 parameters. "VarScan: Conservative" uses the following parameters to stringently call variants: minimum 15510 total reads at the position of interest, minimum of 4 supporting reads to call variant, minimum average 156 quality of 20 , and minimum variant allele frequency of 0.3 . Please see Table 1 for a summary of 157 parameters used for the VarScan comparisons. A template for running VarScan-Cons 158 (Exon_Capture_workflow_v2.pl) is provided at https://sites.google.com/site/cwarden45/scripts.

159 Additional variant callers were also applied to the "No Preprocessing" alignments and the "Full 160 Pipeline" alignments. Freebayes (Garrison \& Marth 2012) was applied to the 1000 Genomes Exon

161 Targeted samples, using default parameters. The Bayesian variant caller in the bcftools function (in the 162 samtools package ( $\underline{\text { Li et al. 2009) }}$ ) was applied to all three datasets, using default parameters (followed by 163 applying vcfutils.pl with a maximum read depth of 200). Unlike GATK and VarScan, samtools has a 164 unique indel format to represent ambiguous indels. Although the ANNOVAR file conversion program can 165 remove all nucleotides that are not part of the indel, the genomic position used to represent this indel is 166 not necessarily the same as GATK and VarScan. For this reason, we only present the samtools SNP 167 results in this manuscript. All analysis was performed on a shared Linux server with concurrent usage 168 (x64, CentOS 5.10, 256 GB RAM, 4 CPU, 8 cores each, 6 x $2.27 \mathrm{GHz}$ processors).

169 Because a publication on the 1000 Genomes exome and targeted exon datasets has not yet been 170 published, we are only reporting variant frequencies for a single chromosome (chr20) in order to comply

171 with 1 KG publication requirements. Thus, only 1,140,996 base pairs of targeted sequence is considered

172 for variant call benchmarks in the 1000 Genomes exome datasets, and only 35,309 base pairs are 173 considered for variant call benchmarks in the 1000 Genomes exon targeted samples. In contrast, the 174 SRP019719 comparisons are genome-wide (with a targeted design covering 46,401,093 base pairs). 
175 Annotating Variants

176 After variants were called, ANNOVAR (Wang et al. 2010) was used to determine the population

177 frequency for each variant, using the summarize_annovar.pl function that was last updated 2/11/2013.

178 Variants were defined as "low frequency" if the 1000 Genomes Project (The 1000 Genomes Project

179 Consortium 2012) frequency and NHLBI Exome Sequencing Project (Fu et al. 2013) frequencies were

180 both less than 0.01 . Variants were defined as "novel" if they were not present in any $1 \mathrm{KG}$ or ESP samples

181 as well as undefined in dbSNP (Sherry et al. 2001). Some rare variants may not truly be truly novel, but

182 this distinction between "novel" and "previously observed" variants should typically be valid. Variants were 183 predicted as damaging if the SIFT ( $\mathrm{Ng}$ \& Henikoff 2003) score greater than or equal to 0.95 or the

184 PolyPhen (Adzhubei et al. 2010) score was greater than or equal to 0.85 (which are the thresholds used 185 by ANNOVAR to flag a variant as damaging, for those two programs). Although population frequency and 186 damaging frequencies can be provided for both SNPs and indels, there were very few indels within on-

187 target regions for the targeted exon samples (so, ANNOVAR characterization is only present for SNPs).

188 ANNOVAR also provides a common format that can compare all variant callers (and it allows easy 189 determination of variants included within the targeted exon panel), so all between-sample comparisons 190 were performed using the ANNOVAR exome summary table (except for the SNP chip comparison, which 191 uses the genome summary table).

192 RESULTS

193 Replication of GATK and VarScan Variant Calls Among Technical Replicates

194 There were two 1000 Genomes (1KG) individuals that had two targeted exon samples (NA18637: 195 SRR013654 + SRR013709, and NA18510: SRR017908 + SRR018122). We treated these samples as

196 technical replicates in order to assess the reproducibility of variant calls from GATK and VarScan. All 197 samples contained Illumina GAll sequencing reads from the Broad Institute (Table S1). The SNP 198 concordance between samples was clearly higher for NA18637 than NA18510 (Figure S1A). These

199 trends hold true when only coding variants were considered, either for all genes (Figure S1B) or only 200 coding variants within targeted genes (Figure S1C). In fact, the concordance rate increases when 201 focusing on coding variants. Similar statistics were provided for indels (Figure S2), but the sample size 202 was too small to compare indels with more focused variant sets. It is unlikely that one NA18510 sample 
203 was simply mislabelled: the concordance between exome and targeted exon samples was more similar

204 (Table S4 and S5), and we would expect lower concordance between two random individuals. One

205 possible explanation is that sample SRR017908 had 36 base pair reads (instead of 76 base pair reads),

206 which could explain the lower concordance among the NA18510 samples (which have varying read

207 lengths, unlike the NA18637 samples). Also, it is worth noting that the concordance of indel calls was

208 better for the GATK UnifiedGenotyper (and usually VarScan) than the GATK HaplotypeCaller (Figure S2),

209 and this was true for both samples. This may corroborate the conclusions of a previous study indicating

210 an increased false discovery rate causes the GATK HaplotypeCaller to produce a larger number of novel

211 variants (Lescai et al. 2014).

\section{GATK and VarScan show Similar Reproducibility Among Validated and SNP Chip Variants}

213 The recovery rate for validated SNPs in 1KG samples was similar for each variant calling pipeline

214 (Figure 1, Table S6). Statistics are only provided for the $1 \mathrm{KG}$ exome samples, because no validated

215 SNPs occur in the targeted regions for the targeted exon samples (for the samples selected for this study).

216 Out of the total 35 validated SNPs present among the 12 exome samples, recovery rates varied between

$21780-94 \%$. VarScan-Default had the highest overall sensitivity and VarScan-Cons had the lowest overall

218 sensitivity. The pre-processing steps had little impact on the results: in fact, the only effect was that base

219 recalibration caused one fewer validated SNP to be recovered when using the GATK UnifiedGenotyper.

220 Unfortunately, this is a limited number of validated SNPs, so it is difficult to say how closely these are tied

221 to the true sensitivity rates.

222 SNP chips can also be used to assess sensitivity for variant lists. However, it should be noted

223 that these will mostly be common SNPs, which importantly means that recovery of SNP array variants

224 may not represent the accuracy of rare variant calls. Nevertheless, it is useful to see how the SNP chip

225 recovery compared to the validated SNPs and the total SNP calls. In most cases, all variant calling

226 algorithms could recover $>85 \%$ of Omni SNP chip variants from the 1000 Genomes exome samples

227 (Figure 2A). Similarly, we compared variant calls for a subject from the SRP019719 dataset that had both

228 Illumina exome and SNP chip data: again, the majority (88-96\%) of SNP chip variants were recovered in

229 the paired exome dataset (Figure 2B, Table S7). In both cases, recovery was restricted to SNP chip

230 variants within targeted regions for the exome sample. It is also worth noting that SNP chip alleles 
231 matching the reference sequence are not considered, and the recovery rate would of course be higher if

232 these sites were considered. Unlike the validated variants (Figure 1), VarScan (using default parameters)

233 no longer had the highest sensitivity; instead, the average recovery of SNP chip variants was slightly

234 higher for GATK. In short, we believe that all variant calling strategies show a similar recovery rate for

235 validated SNPs and SNP chip variants, with a false negative rate for these common variants likely being

236 less than $15 \%$.

\section{False Positives are Enriched for Novel Variants}

238 The recovery of targeted exon variants (within the set of targeted genes) among the exome 239 samples was also typically quite high (Figure 3A). The main exception was for variants called using 240 VarScan with default parameters, and this was also true for the VarScan calls with the p-value filter (to a

241 lesser extent). In this case, it is important to note that the targeted exon calls are not truly a gold standard.

242 In other words, the lower recovery rate can be due to a high false discovery rate among the targeted exon

243 variant calls. For example, position 17933286 on chromosome 20 for individual NA18566 was covered by

244189 reads in the targeted exon sample and 109 reads in the exome sample: $90 \%$ of reads match the

245 reference sequence in the targeted exon sample and 99\% percent of the reads match the reference

246 sequence in the exome sample. Using the default parameters, VarScan calls a variant "G" allele in the

247 targeted exon sample (which is present in 12/189 reads). However, this is likely a normal diploid individual

248 with the true genotype of $\mathrm{T} / \mathrm{T}$ at this position, with deviations from the reference sequence that are

249 probably due to technical error (where the proportion of errors found at a particular site can randomly

250 fluctuate between samples). While this specific variant may serve a useful conceptual example, it is

251 important to identify subsets of variants are likely to drive the lower recovery rate among the VarScan-

252 Default variant calls.

253 If variant lists are confined to previously observed variants (present in the 1000 Genomes Project,

254 Exome Sequencing Project, or dbSNP), then recovery of targeted exon VarScan-Default SNPs are

255 considerably improved. For example, VarScan calls with the $p$-value filter were now comparable to the

256 other variant callers (Figure 3B). An increased false discovery rate has been observed for loss of function

257 variations due to the presence of uniform sequencing errors and non-random distribution of natural

258 variation (MacArthur et al. 2012), and we believe a similar concept applies more broadly to novel variants. 
259 Accordingly, the recovery rate was extremely low for novel variants called using VarScan with default 260 parameters (Table S8). There were no novel VarScan-Cons variants called within the coding regions of

261 chromosome 20, but it was likely difficult to call true novel variants given that variants called by the 1000

262 Genomes Project are defined as previously observed in our analysis. The recovery rate was also

263 relatively low for other variant callers, but there are only a small number of novel variants occurring within

264 targeted regions of chr20 for most of the lists of variant calls.

265

One sample in the SRP019719 dataset underwent exome sequencing as well as amplicon

266 sequencing for a selected number of variants. Although limited to a single sample, this comparison was

267 important because the amplicon dataset contained more novel variants than present within 1000

268 Genomes targeted exon genes on chromosome 20. The amplicon comparison showed a universal

269 decrease in the recovery of novel variants (Figure S3), although it should be noted that the overall

270 recovery rate was also lower than the array-based enrichment samples. This is most likely because

271 duplicate reads were not removed, and errors introduced during PCR amplification increased the total

272 number of false positives in the amplicon dataset. For example, the targeted variants were located

273 towards the middle of each amplicon, but sequencing errors may be more frequent towards the end of the

274 amplicons. This expectation of an increased false discovery rate is supported by the fact that the number

275 of SNPs called in the amplicon dataset (Table S9) is much higher than the number of variants that were

276 targeted for validation (O'Rawe et al. 2013). Accordingly, the novel variant frequency is much higher for

277 the amplicon datasets than the exome and targeted exon datasets (Table S10). Of course, changing

278 variant calling criteria (such as imposing stricter coverage requirements) will affect the number of false

279 positives (and thus the novel variant rate), so this result should not be interpreted as an indication that it is

280 impossible to get high quality results from the amplicon dataset. Instead, this result emphasizes that

281 benchmarks will vary with different target designs, while still demonstrating the general increase in false

282 discovery rate for novel variants (although the extent of enrichment can vary with target design and variant 283 caller).

284 In the example of the specific variant at position 17933286 on chromosome 20, there was a very 285 small proportion of reads containing the variant allele. If the density distributions of novel and previously286 observed variations are compared, it is clear that most of the novel variants called by VarScan-Default had 287 less than $20 \%$ of reads containing the variant allele (Figure 4 and S4). We expect the most conservative 
288 aspect of VarScan-Cons is the requirement that variants be present in at least $30 \%$ of reads. To test this

289 hypothesis, we calculated the recovery rate for targeted exon VarScan-Default variants with varying

290 thresholds for the percentage of reads containing the variant allele as well as the minimum number of

291 reads with the variant allele. There were no novel variants called by VarScan-Cons and all novel variants

292 supported by less than $30 \%$ of reads had significantly lower recovery than previously-observed variants

293 with comparable support (Table S11, Figure S5). Recovery rates among previously observed variants

294 droped when a threshold of $50 \%$ supporting reads was used because this threshold splits the

295 heterozygous peak in half (increasing the likelihood of encountering variants that marginally meet the

296 criteria in one dataset but marginally do not meet the criteria in the other dataset). In short, novel variants

297 often have an increased false discovery rate because the low likelihood of sequencing error occurring for

298 the same nucleotide at the same position of a naturally occurring variation, where most novel variants

299 (defined using sufficiently liberal criteria) fit the model of a sequencing error that will be observed most

300 frequently in variants with small percentages of supporting reads.

301 The difference in density distributions was most clear for variant with less than $20 \%$ supporting

302 reads, but the entire peak of variants with less than $30 \%$ supporting reads violates an assumption a diploid

303 human genome. In other words, reads matching the reference genome were not called as variants, so the

304 homozygous wild-type allele should not have a peak in the density distribution. This is the basis of

305 selecting this threshold for VarScan-Cons. As expected, there were only two peaks for the density

306 distribution of supporting reads for VarScan-Cons (Figure 5). This was seen most clearly for the

307 SRP019719 exome data, where there were a sufficiently large number of novel variants to define clean

308 density distributions. Importantly, this was also true after removing low-quality GATK UnifiedGenotyper

309 and HaplotypeCaller (Figure 5), which indicates that most variants with less than $30 \%$ supporting reads

310 were also removed using this independent variant calling strategy. This particular supporting read

311 threshold was specifically designed with a diploid organism in mind, but technical errors should always be

312 enriched among all variants with low percentages of supporting reads. Therefore, visualization of density

313 distributions for supporting read frequencies of novel variants can be a useful strategy for comparing

314 variants lists for any organism.

\section{Estimation of Accuracy for GATK and VarScan Variant Calls}


Although 1KG samples were selected on the basis of having some validated positive controls, it is

317 useful to have quality control metrics that will be correlated with the true sensitivity and specificity for a

318 given variant caller. Each strategy has notable caveats for interpretation, but we think it is useful to have

319 quality control metrics that can be used to select an optimal variant caller for a given dataset.

First, we assume that the total number of variants is correlated with sensitivity. Of course, the

321 accuracy of this assumption would depend upon the false discovery rate for the variant caller.

322 Nevertheless, we believe that the total number of called variants is a useful benchmark to compare variant

323 callers. SNP and indel counts are shown for $1 \mathrm{KG}$ exome and targeted exon samples in Figure 6 . In most

324 cases, the pre-processing pipeline had a minimal effect on the size of the resulting list of SNPs, with the

325 notable exception of the GATK UnifiedGenotyper (although the impact was significantly decreased for

326 high-quality SNPs). Similarly, the overlap was strong for variants called without these extra pre-

327 processing steps (Figure S6 and S7). However, it is difficult to tell how common this trend is for all

328 datasets: for example, the exome samples from SRP019719 did not show this same difference (Figure

329 S8) and instead showed a relatively greater difference in the number of SNPs called by VarScan with

330 default parameters. The size of the SNP lists clearly varied with different VarScan parameters: the p-value

331 threshold considerably decreased the number of SNPs called, the conservative parameter set was even

332 more restrictive, and both results run with non-default settings produced significantly fewer SNPs than

333 GATK. Among the high-quality calls, the number of SNPs was similar for GATK HaplotypeCaller versus

334 GATK UnifiedGenotyper. The relative number of indels varied from the relative number of SNP calls:

335 however, there were only a limited number of indel calls on chr20 for the targeted exon panel, so we think

336 the number of $1 \mathrm{KG}$ exome indel calls were more reliable for assessing general trends. There was a

337 modest but noticeable increase in the number of indels called if the indel realignment was run, and the

338 GATK HaplotypeCaller produced a much larger number of indels than the GATK UnifiedGenotyper.

339 Similar to the SNP calls, VarScan produces fewer indel calls when using more stringent parameters.

Second, we assume that most variants should have been previously observed in large scale

341 sequencing project (like the 1000 Genomes Project and the Exome Sequencing Project) and variant

342 databases (like dbSNP), and that an over-representation of variants of unknown frequencies (e.g. novel

343 variants) should correspond to an increased false discovery rate. Of course, some novel variants will in

344 fact be accurate and the proportion of true novel variants will vary with demographics (subjects from 
345 ethnicities that have been characterized in greater detail will likely show fewer novel variants, and vice

346 versa). However, some proportion of novel variants are clearly unacceptable: for example, more than

$34750 \%$ of the variants called by VarScan (using default parameters) were novel variants, and the majority of

348 these novel variants were predicted to be damaging (Figure 7 and S9). In contrast, only a minority of

349 variant calls $(0.4-9.7 \%$ for $1 \mathrm{KG}$ exome; $0.8-4.2 \%$ for $1 \mathrm{KG}$ targeted exon; $0.1-2.1 \%$ for SRP019719 exome

350 samples) were known to be present at low frequency (<1\%; see Methods) in the overall population, and

351 this was true for all samples using all variant calling strategies. Importantly, this abnormally high

352 proportion of novel variants matched the considerably decreased recovery rate between exome and

353 targeted exome datasets, which we have been shown to be caused by a very low recovery rate among

354 novel variants (Figure 3, Tables S8 and S11).

355 Most GATK variant lists had a similar proportion of novel variants, except when the GATK

356 UnifiedGenotyper was run without quality score recalibration in the 1000 Genomes samples (Figure 7).

357 However, trend doesn't apply to the SRP019719 samples (Figure S9), emphasizing that there were other

358 factors that can influence these results. The pre-processing steps had a modest impact on the VarScan

359 frequencies, but the VarScan parameters had a very strong impact on the results. Namely, the frequency

360 of novel variants was extremely high when running VarScan with default parameters, which corresponded

361 to a decrease in the overall recovery rate rate (Figure 3). However, the distribution for VarScan variants

362 called with conservative parameters looked very similar to the GATK distributions, and these variants had

363 a high recovery rate between the 1KG exome and targeted exon samples (Figure 3).

ANNOVAR can also annotate variant frequencies for indels. However, small indels have not been

365 characterised as well as SNPs (for example, there are considerably fewer indels in dbSNP (Sherry et al.

366 2001), compared to SNPs) and damaging predictions focus primarily on SNPs. Additionally, there are

367 almost no indels in the coding regions of chromosome 20 for the 1000 Genomes targeted exon dataset.

368 Nevertheless, Figure S10 shows the variant frequencies for indel calls in the 1KG and SRP019719 exome

369 datasets. Similar to the SNP distributions, VarScan-Cons contained the least number of novel indels

370 (among the VarScan comparisons), which was likely associated with a lower false discovery rate. Also,

371 novel indels were more common in GATK HaplotypeCaller variants than GATK UnifiedGenotyper variants.

372 Arguably, this could indicate that the higher number of indels called by the HaplotypeCaller was also

373 associated with a higher false discovery rate, but the variant frequencies alone are not sufficient to prove 
374 this to be the case because there may have been technical limitations in discovering the indels without

375 using the GATK HaplotypeCaller. However, lower concordance for HaplotypeCaller indels between

376 technical replicates (Figure S2) supports the hypothesis that the HaplotypeCaller indels had a higher false

377 discovery rate. Similarly, recovery of indels was higher for UnifiedGenotyper variants than

378 HaplotypeCaller variants in the SRP019719 sample with both exome and amplicon sequencing data

379 (Figure S11). Interestingly, VarScan-Cons had the highest recovery rate of amplicon sequencing indels for 380 that sample. Although the number of samples available for useful comparisons was limited, these results

381 provide evidence from two independent cohorts that the GATK HaplotypeCaller indels are less

382 reproducible than GATK UnifiedGenotyper indels.

\section{Overlap of High-Quality GATK and VarScan Variant Calls}

384 Given the previous comparisons, we believe the highest quality variant calls can be made by 385 excluding GATK variants with LowQual flags (e.g. UG-HQ and HC-HQ) and using the conservative 386 parameters defined in this manuscript when running VarScan (e.g. VarScan-Cons). Because the number 387 of variants called increased when running the indel realignment and quality score recalibrator steps, we 388 compared coding variant lists produced using variant callers with "Full Pipeline" pre-processing steps. In 389 the 1000 Genomes samples, the variant caller overlap was quite high (Figure 8 and Figure S12). For 390 example, all SNPs called using VarScan-Cons were also called using the GATK HaplotypeCaller.

391 Additionally, all indels called using VarScan-Cons were also called by either the GATK HaplotypeCaller or 392 the GATK UnifiedGenotyper. Because only chromosome 20 variants can be reported for the 1000 393 Genomes dataset in this manuscript, it may also be useful to see the overlap for the genome-wide variant

394 lists from SRP019719 (Figure 8). This time, the VarScan-Cons variants were not completely recovered by 395 using one or both GATK variant callers, but there was still only a minority of variants that were not 396 represented in either list of GATK variants (2.9\% of VarScan-Cons SNPs and $1.5 \%$ of VarScan-Cons

397 indels). This emphasizes that VarScan-Cons calls robust variants that would almost always be called 398 when using GATK.

399 The high recovery of VarScan-Cons variants may result from high specificity with decreased 400 sensitivity, which is potentially the biggest drawback to this strategy. The degree to which VarScan-Cons 401 recovers the set of high-quality GATK variants varied between datasets. Among the 1000 Genome 
402 variants on chromosome $20,87 \%$ of UnifiedGenotyper SNPs and $88 \%$ of HaplotypeCaller SNPs were also

403 called by VarScan-Cons in the exome samples. For the targeted exon samples, $62 \%$ of UnifiedGenotyper

404 SNPs and $68 \%$ of HaplotypeCaller SNPs were called by VarScan-Cons in the targeted exon sample;

405 similarly, $69 \%$ of UnifiedGenotyper indels but only $38 \%$ of HaplotypeCaller indels were also called by

406 VarScan-Cons in the $1 \mathrm{KG}$ exome samples. The low indel overlap was likely due to the small number of

407 coding indels on chromosome 20. Accordingly, the SRP019719 exome samples show similar statistics for

408 SNP overlap (84\% recovery for UnifiedGenotyper SNPs, 86\% recovery for HaplotyperCaller SNPs) but

409 much better results for UnifiedGenotyper indels ( $81 \%$ recovery). There was still only $51 \%$ recovery for

410 HaplotypeCaller indels among VarScan-Cons indels, but it is possible that the indels uniquely called by

411 GATK have a higher false discovery rate. An increased false discovery rate for GATK HaplotypeCaller

412 indels has been previously reported (Lescai et al. 2014) and this conclusion is supported by evidence from

413 this study (Figures S2, S10-S11).

\section{Run-Times Scale Differently for GATK versus VarScan}

Figure $\mathrm{S} 13$ shows run-times for the entire variant calling pipeline for the $1 \mathrm{KG}$ samples. The run-

416 time for each step is reported in Table S12-S14, with the average run-times for the variant calling step

417 itself shown in Table S15 (for the "Full Pipeline" samples). Among the targeted exon samples ( $n=14$ ), the

418 GATK UnifiedGenotyper had the longest run-time (Figure S13A). However, the run-time for all samples

419 was less than 5 hours, so run-time was not a severe limiting factor for either VarScan or GATK (for

420 targeted exon samples, compared to the exome samples). Adding a p-value filter did not significantly

421 affect the run-time for VarScan; in fact, the run-time for VarScan was essentially the same regardless of

422 what parameters are used for analysis. In contrast, the $1 \mathrm{KG}$ exome samples showed a wide range of run-

423 times (Figure S13B), with the HaplotypeCaller having a very noticeably longer run-time compared to the

424 other pipelines. Quality score recalibration also caused a noticeable increase in run-time. As expected,

425 the newest version of GATK (with or without read reduction) decreased the run-time for GATK

426 HaplotypeCaller (Table S15-S17). However, the run-time was always shorter for VarScan compared to

427 GATK.

For the SRP019719 exome samples, the most obvious trend was the run-time for the GATK

429 HaplotypeCaller was considerably longer when running base recalibration without prior indel realignment 
430 (Figure S14). Likewise, population frequencies among variants (Figure S9) indicate that the variants

431 called using base recalibration alone were probably not reliable (for these particular samples), but the

432 variants called using the full pipeline (indel realignment + base recalibration) were more likely to have a

433 lower false discovery rate (similar to the "No Preprocessing" and "Realign Only" results). Two factors

434 mostly likely caused this increased-run time are 1) quality scores were on a different scale than the $1 \mathrm{KG}$

435 samples and 2) the servers running the variant callers had considerable concurrent usage (where

436 variability in usage may explain why the run-times for some samples were much worse than other

437 samples). More specifically, the quality scores were identified as miscoded by GATK, and it appears that

438 the quality scores could not be successfully fixed at the base quality recalibration step (see Text S1 and

439 S2 for more details). In contrast, the miscoded quality scores could be successfully shifted by GATK prior

440 to quality score recalibration or at the variant calling step (if neither indel realignment nor quality score

441 recalibration was run). Therefore, we do not expect this trend to apply to most samples that are

442 processed in accordance to GATK "best practices".

Run-times can also be affected a running joint variant caller instead of running variant callers

444 separately for each sample. One potential advantage to this strategy is that it can increase sensitivity in

445 cohorts of related individuals (Nielsen et al. 2011). One disadvantage is that joint variant calling can have

446 substantial memory requirements for large cohorts. However, joint variant calling could be successfully

447 run on all the groups of samples in this study, with a substantial decrease in run times for the GATK joint

448 variant caller (Table S16). In contrast, joint variant calling considerably increased the runtime for VarScan,

449 but the total run-time for single-sample VarScan calls was still less than the run-time for the joint variant

450 calls in GATK (Table S16).

\section{Comparison to Other Variant Calling Benchmarks}

452 In order to provide a way to quickly compare VarScan-Cons results to other variant callers (with 453 other pre-processing pipelines), the Genome Comparison \& Analytic Testing (GCAT) website was used to 454 compare six sets of variants calls for a standard sample that has been characterized by the Genome in a 455 Bottle Consortium (Zook et al. 2014). This sample contained 100 base pair Illumina reads for a 150x 456 Exome sample (making most comparable to the samples in this study). This is not the only available tool 457 that can benchmark variant caller algorithms (Nevado \& Perez-Enciso 2014; Talwalkar et al. 2014), but it 
458 was selected based upon the ability to compare a targeted sequencing sample without the use of

459 simulated data (similar to the design of the current study). Links to reports for each variant list are

460 provided in Table S18. Although the presence of a single (albeit well-characterized) sample is a limitation

461 to the strategy, the GCAT report shows results that complement and enhance this study. For example,

462 VarScan-Cons showed a decrease in sensitivity when recovering Omni SNP array genotypes but also

463 showed the highest specificity and precision rate, when compared to variant calls with no pre-processing

464 steps as well as with the full pre-processing pipeline (Figure S15). The GCAT reports show variant counts

465 and overlap of variants between variant callers that match what is expected from this study (Figure S16),

466 although it is important to note that these are genome-wide counts (similar to Figure 6 and S8) whereas

467 most statistics are providing in this study are for coding variants (such as Figure 8-9). In other words,

468 VarScan-Cons shows similar on-target variant calls to the high-quality GATK variant lists, but GATK made

469 more off-target calls in lower coverage regions. Importantly, the GCAT report also showed the transition to

470 transversion (Ti/Tv) ratio for SNPs for novel as well as common SNPs. The average value for Ti/Tv is

471 between 2.1 and 2.8, so lower ratios can be an indication of an increased false discovery rate. As we

472 would expect from the reproducibility results from this experiment, novel VarScan-Cons variants showed a

473 considerably higher Ti/Tv ratio (and therefore considerably lower false discovery rate) that was more

474 similar to the expected value than the novel high-quality GATK variants (Figure S17).

\section{Application of QC Metrics to Other Variant Callers}

Although we were primarily interested in comparing VarScan (with various sets of parameters) to

477 GATK, we also examined similar metrics for other variant callers. Given that the distribution of rare and

478 novel variant frequencies was similar for the 1000 Genomes targeted exon and exome datasets, we used

479 the targeted exon samples to test additional variant callers. More specifically, we tested freebayes

480 (DePristo et al. 2011) and samtools (Li et al. 2009), using the "No Preprocessing" and "Full Pipeline"

481 alignments. It is clear that freebayes yields too many novel variants (Figure S18), so it was not tested on

482 any further datasets. Of course, changes in parameters and/or downstream filtering of variants may result

483 in a decrease in the frequency of novel variants; however, these two lists of variants (called with default

484 parameters) are meant to serve as examples of how the quality control metrics in this dataset could be

485 applied more broadly. Interestingly, the pre-processing steps appeared to have minimal effect on the 1000

486 Genomes targeted exon and exome datasets, but running the "Full Pipeline" for pre-processing 
487 considerably decreased the proportion of novel variants for samtools variant calls in the SRP019719

488 exome dataset (Figure S18). Most samtools variants overlaped VarScan-Cons variants as well as high-

489 quality GATK UnifiedGenotyper and HaplotypeCaller variants (Figure S19).

490 The ANNOVAR frequency plots (like those shown in Figure 7, S9, and S18) are a useful metric,

491 but they only show average trends across the dataset and do not show how the frequency of novel

492 variants compares to the total number of variants called. If the number of conditions is reduced to only

493 those using the "Full Pipeline" for pre-processing, then one can visualize the comparison between variant

494 callers (Figure 9). There was more variability in the proportion of novel variants for the targeted exon

495 dataset (compared to either exome dataset), but this may relate to the lower number of total SNPs and/or

496 the lower on-target coverage (Table S1-S3). While it is not necessarily safe to assume that the false

497 discovery rate substantially varies when the proportion of novel variants is between 0 and $6 \%$ (which is the

498 observed range for these variant callers in the exome datasets), the proportion of novel variants is

499 universally low for all VarScan-Cons calls in all three datasets while there is more variability in the

500 proportion of novel variants in the GATK and samtools variant calls. VarScan-Cons and the high-quality

501 GATK variant calls showed similar distributions of supporting reads (Figure 5), so the larger number of

502 novel variants was not being driven by variants that were extremely likely to be false positives (Figure 4).

503 Nevertheless, there was a decrease in recovery rate (9-20\% for 5-11 UnifiedGenotyper SNPs and 30-50\%

504 for 1-3 HaplotypeCaller SNPs) for a limited number of novel, high-quality GATK variants compared to

505 common, high-quality GATK variants (Table S8, Figure 3), but the VarScan-Cons criteria were so strict that

506 no novel variants were called within targeted exon regions on chromosome 20. Although we cannot make

507 a definitive conclusion using the data in this current study, the difference in the percentage of novel SNPs

508 could be explained if VarScan-Cons had a relatively lower false discovery rate among novel, high-quality

509 variants. This hypothesis can be supported by the observation that novel VarScan-Cons variants showed

510 a substantially higher Ti/Tv ratio that is much more similar to common variants (Figure S17), so it is

511 plausible that novel VarScan-Cons variants indeed have a decreased false discovery rate relative to novel

512 GATK variants for array-based targeted sequencing experiments.

\section{DISCUSSION}


$514 \quad$ Although running VarScan with default parameters (with the functions defined in the Methods

515 section) was shown to result in an unacceptably high false discovery rate (Figure 3), running VarScan with

516 a conservative set of non-standard parameters (referred to as VarScan-Cons in this study) can produce a

517 reliable set of variants with an overall concordance between sequencing technologies that was at least as

518 strong as high-quality GATK variants (Figures 1-3, S1-S3, S11, and S17). Almost all variants called with

519 VarScan-Cons were also called using the GATK HaplotypeCaller or GATK UnifiedGenotyper, with a

520 modest decrease in sensitivity for SNPs (Figure 1-2, S15). However, given that the high-quality GATK

521 variants have a similar overall specificity to VarScan-Cons (Figure 1-3, 7, and S15), increased specificity is

522 one advantage to using GATK HaplotypeCaller or GATK UnifiedGenotyper (Figure 6 and S16). Although

523 less obvious than the VarScan variant lists, the high-quality GATK variant lists consistently show improved

524 recovery rate for targeted exon variants (Figure 3) and lower percentages of novel variants (Figure 7),

525 even without additional pre-processing steps. So, we would recommend VarScan users use the VarScan-

526 Cons parameters and GATK users to filter out variants flagged as low-quality. In all three cases (VarScan-

527 Cons, high-quality GATK HaplotypeCaller, high-quality GATK UnifiedGenotyper), indel realignment and

528 quality score recalibration affected only a minority of variants (Figure S6-S7), so users could arguably skip

529 those pre-processing steps and still get similar results (if a modest decrease in overall validation rate was

530 acceptable). GATK HaplotypeCaller called a substantial number of indels not called using VarScan-Cons

531 (as well as GATK UnifiedGenotyper), even after removing variants that were flagged as low quality.

532 However, it is possible that the GATK HaplotypeCaller indels also have an increased false discovery rate

533 (Figures S2, S10-S11), as reported in (Lescai et al. 2014), so it will be important to see if this is

534 independently validated in other studies.

535 It is important to note that most of the statistics used for calling VarScan variants can be extracted

536 from the .vcf file containing the GATK variants. In other words, users may not need to run both GATK and

537 VarScan. For example, the distributions of supporting read frequencies look very similar for the high-

538 quality GATK variants and the VarScan-Cons variants (Figure 5), so excluding GATK variants flagged as

539 low-quality can already produce results that are more similar to VarScan-Cons. This study presents some

540 limited evidence that VarScan-Cons variants may have a lower false discovery rate among novel variants

541 in array-based enrichment designs, compared to the high-quality GATK variant calls (Table S8 and Figure

542 S17). So, GATK users may be able to improve the accuracy of their novel variant calls by imposing 
543 stricter requirements on frequency of supporting reads, number of supporting reads, and/or read depth.

544 This is important because high-throughput sequencing studies often emphasize novel, rare variants as

545 interesting candidates (Worthey 2013). Based upon the results of this study, we expect that variant calling

546 comparisons that only focus on complete sets of variant calls (where most variants are likely to be

547 common variants) may give false confidence in the accuracy of variant calls for novel variants. However,

548 additional evidence is necessary to confirm that VarScan-Cons defines more accurate novel SNPs than

549 the high-quality GATK variants. Nevertheless, we believe that this study provides good evidence that

550 users should be suspicious of lists of variants with substantially increased novel variant frequencies, such

551 as those with novel variant frequencies of $50 \%$ or higher (like VarScan-Default).

552 This study focused on variant calling in (most likely) normal human samples due to the availability

553 of a large amount of public validation data. However, the strategies described in this study may not apply

554 equally well in all circumstances. For example, sometimes variants may be present in a minority of cells in

555 a sample (such as a heterogeneous tumor), and it may not be safe to make assumptions about the ploidy

556 of the sample (which might affect the usefulness of the GATK HaplotypeCaller, for example). In fact, the

557 results of this study emphasize the need to have specialized experimental protocols (such as circle

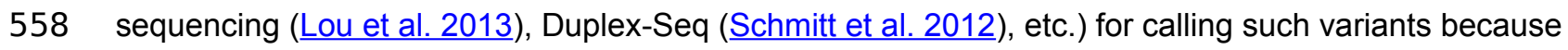

559 variants with low numbers of supporting reads show an extreme decrease in recovery rate between

560 different targeted sequencing designs (Figure 4 and S4, and Table S8 and S11). Likewise, somatic variant

561 calling is also an area of interest (Roberts et al. 2013; Wang et al. 2013; Xu et al. 2014) that would

562 typically utilize different variant calling tools. Additionally, the indel metrics in this study only apply to small

563 indels: large indels and structural variants require a different set of algorithms. Nevertheless, we think the

564 strategies described in this study (comparing the proportion of novel variants, using filters for high-quality

565 variants, etc.) can be useful to help other scientists prioritize variant calling strategies for their own data.

\section{ACKNOWLEDGEMENTS}

567 We would like to thank Laura Clarke for providing technical assistance with the 1000 Genomes data,

568 Jason O'Rawe and Gholson Lyon for answering questions about the O'Rawe et al. 2013 exome dataset

569 and providing access to the Human610 SNP chip data and providing helpful comments to improve this 
570 manuscript, and Illumina technical assistance for answering questions about the Illumina SNP chips. We 571 would also like to thank Han Fang and an anonymous reviewer for helpful comments. 
573

574

575

576

577

578

579

580

581

582

583

584

585

586

587

588

589

590

591

592

593

594

595

596

597

598

599

600

601

602

603

604

605

606

607

608

609

610

611

612

613

614

615

616

617

618

619

620

621

622
Adzhubei IA, Schmidt S, Peshkin L, Ramensky VE, Gerasimova A, Bork P, Kondrashov AS, and Sunyaev SR. 2010. A method and server for predicting damaging missense mutations. Nat Meth 7:248-249.

Bauer D. 2011. Variant calling comparison CASAVA1.8 and GATK. Nature Precedings http://dx.doi.org/10.1038/npre.2011.6107.1.

Blankenberg D, Kuster GV, Coraor N, Ananda G, Lazarus R, Mangan M, Nekrutenko A, and Taylor J. 2001. Galaxy: A Web-Based Genome Analysis Tool for Experimentalists. Current Protocols in Molecular Biology: John Wiley \& Sons, Inc.

Boland J, Chung C, Roberson D, Mitchell J, Zhang X, Im K, He J, Chanock S, Yeager M, and Dean M. 2013. The new sequencer on the block: comparison of Life Technology's Proton sequencer to an Illumina HiSeq for whole-exome sequencing. Human Genetics 132:1153-1163.

Carson A, Smith E, Matsui H, Braekkan S, Jepsen K, Hansen J-B, and Frazer K. 2014. Effective filtering strategies to improve data quality from population-based whole exome sequencing studies. BMC Bioinformatics 15:125.

Cheng AY, Teo Y-Y, and Ong RT-H. 2014. Assessing single nucleotide variant detection and genotype calling on whole-genome sequenced individuals. Bioinformatics 30:1707-1713.

DePristo MA, Banks E, Poplin R, Garimella KV, Maguire JR, Hartl C, Philippakis AA, del Angel G, Rivas MA, Hanna M, McKenna A, Fennell TJ, Kernytsky AM, Sivachenko AY, Cibulskis K, Gabriel SB, Altshuler D, and Daly MJ. 2011. A framework for variation discovery and genotyping using next-generation DNA sequencing data. Nat Genet 43:491-498.

Fu W, O/'Connor TD, Jun G, Kang HM, Abecasis G, Leal SM, Gabriel S, Altshuler D, Shendure J, Nickerson DA, Bamshad MJ, Project NES, and Akey JM. 2013. Analysis of 6,515 exomes reveals the recent origin of most human proteincoding variants. Nature 493:216-220.

Garrison E, and Marth G. 2012. Haplotype-based variant detection from short-read sequencing. arXiv preprint arXiv:1207.3907 [q-bio.GN].

Giardine B, Riemer C, Hardison RC, Burhans R, Elnitski L, Shah P, Zhang Y, Blankenberg D, Albert I, Taylor J, Miller W, Kent WJ, and Nekrutenko A. 2005. Galaxy: A platform for interactive large-scale genome analysis. Genome Research 15:1451-1455.

Goecks J, Nekrutenko A, Taylor J, and Team TG. 2010. Galaxy: a comprehensive approach for supporting accessible, reproducible, and transparent computational research in the life sciences. Genome Biology 11:R86.

Koboldt DC, Chen K, Wylie T, Larson DE, McLellan MD, Mardis ER, Weinstock GM, Wilson RK, and Ding L. 2009. VarScan: variant detection in massively parallel sequencing of individual and pooled samples. Bioinformatics 25:2283-2285.

Koboldt DC, Zhang Q, Larson DE, Shen D, McLellan MD, Lin L, Miller CA, Mardis ER, Ding L, and Wilson RK. 2012. VarScan 2: Somatic mutation and copy number alteration discovery in cancer by exome sequencing. Genome Research 22:568-576.

Lam HYK, Pan C, Clark MJ, Lacroute P, Chen R, Haraksingh R, O'Huallachain M, Gerstein MB, Kidd JM, Bustamante CD, and Snyder M. 2012. Detecting and annotating genetic variations using the HugeSeq pipeline. Nat Biotech 30:226-229.

Leinonen R, Akhtar R, Birney E, Bower L, Cerdeno-Tárraga A, Cheng Y, Cleland I, Faruque N, Goodgame N, Gibson R, Hoad G, Jang M, Pakseresht N, Plaister S, 
627

628

629

630

631

632

633

634

635

636

637

638

639

640

641

642

643

644

645

646

647

648

649

650

651

652

653

654

655

656

657

658

659

660

661

662

663

664

665

666

667

668

669

670

671

672

673

674

675
Radhakrishnan R, Reddy K, Sobhany S, Ten Hoopen P, Vaughan R, Zalunin V, and Cochrane G. 2011. The European Nucleotide Archive. Nucleic Acids Research 39:D28-D31.

Lescai F, Marasco E, Bacchelli C, Stanier P, Mantovani V, and Beales P. 2014. Identification and validation of loss of function variants in clinical contexts. Molecular Genetics \& Genomic Medicine 2:58-63.

$\mathrm{Li} \mathrm{H}$, and Durbin R. 2009. Fast and accurate short read alignment with BurrowsWheeler transform. Bioinformatics 25:1754-1760.

Li H, Handsaker B, Wysoker A, Fennell T, Ruan J, Homer N, Marth G, Abecasis G, Durbin R, and Subgroup GPDP. 2009. The Sequence Alignment/Map format and SAMtools. Bioinformatics 25:2078-2079.

Li J, Doyle MA, Saeed I, Wong SQ, Mar V, Goode DL, Caramia F, Doig K, Ryland GL, Thompson ER, Hunter SM, Halgamuge SK, Ellul J, Dobrovic A, Campbell IG, Papenfuss AT, McArthur GA, and Tothill RW. 2014. Bioinformatics Pipelines for Targeted Resequencing and Whole-Exome Sequencing of Human and Mouse Genomes: A Virtual Appliance Approach for Instant Deployment. PLoS ONE 9:e95217.

Linderman M, Brandt T, Edelmann L, Jabado O, Kasai Y, Kornreich R, Mahajan M, Shah H, Kasarskis A, and Schadt E. 2014. Analytical validation of whole exome and whole genome sequencing for clinical applications. BMC Medical Genomics 7:20.

Liu X, Han S, Wang Z, Gelernter J, and Yang B-Z. 2013. Variant Callers for NextGeneration Sequencing Data: A Comparison Study. PLoS ONE 8:e75619.

Lou DI, Hussmann JA, McBee RM, Acevedo A, Andino R, Press WH, and Sawyer SL. 2013. High-throughput DNA sequencing errors are reduced by orders of magnitude using circle sequencing. Proceedings of the National Academy of Sciences.

MacArthur DG, Balasubramanian S, Frankish A, Huang N, Morris J, Walter K, Jostins L, Habegger L, Pickrell JK, Montgomery SB, Albers CA, Zhang ZD, Conrad DF, Lunter G, Zheng $H$, Ayub Q, DePristo MA, Banks E, Hu M, Handsaker RE, Rosenfeld JA, Fromer M, Jin M, Mu XJ, Khurana E, Ye K, Kay M, Saunders GI, Suner M-M, Hunt T, Barnes IHA, Amid C, Carvalho-Silva DR, Bignell AH, Snow C, Yngvadottir B, Bumpstead S, Cooper DN, Xue Y, Romero IG, Consortium GP, Wang J, Li Y, Gibbs RA, McCarroll SA, Dermitzakis ET, Pritchard JK, Barrett JC, Harrow J, Hurles ME, Gerstein MB, and Tyler-Smith C. 2012. A Systematic Survey of Loss-of-Function Variants in Human Protein-Coding Genes. Science 335:823-828.

McKenna A, Hanna M, Banks E, Sivachenko A, Cibulskis K, Kernytsky A, Garimella K, Altshuler D, Gabriel S, Daly M, and DePristo MA. 2010. The Genome Analysis Toolkit: A MapReduce framework for analyzing next-generation DNA sequencing data. Genome Research 20:1297-1303.

Narzisi G, O'Rawe JA, lossifov I, Fang H, Lee Y-h, Wang Z, Wu Y, Lyon GJ, Wigler M, and Schatz MC. 2014. Accurate detection of de novo and transmitted INDELS within exome-capture data using micro-assembly. bioRxiv 001370.

Nevado B, and Perez-Enciso M. 2014. Pipeliner: software to evaluate the performance of bioinformatics pipelines for next-generation resequencing. Mol Ecol Resour [Epub ahead of print].

$\mathrm{Ng} \mathrm{PC}$, and Henikoff S. 2003. SIFT: predicting amino acid changes that affect protein function. Nucleic Acids Research 31:3812-3814.

Nielsen R, Paul JS, Albrechtsen A, and Song YS. 2011. Genotype and SNP calling from next-generation sequencing data. Nat Rev Genet 12:443-451.

O'Rawe J, Jiang T, Sun G, Wu Y, Wang W, Hu J, Bodily P, Tian L, Hakonarson H, Johnson WE, Wei Z, Wang K, and Lyon G. 2013. Low concordance of multiple 
676

677

678

679

680

681

682

683

684

685

686

687

688

689

690

691

692

693

694

695

696

697

698

699

700

701

702

703

704

705

706

707

708

709

710

711

712

713

714

715

716

717

718

719

720

721

722

723

724 variant-calling pipelines: practical implications for exome and genome sequencing. Genome Medicine 5:28.

Pabinger S, Dander A, Fischer M, Snajder R, Sperk M, Efremova M, Krabichler B, Speicher MR, Zschocke J, and Trajanoski Z. 2014. A survey of tools for variant analysis of next-generation genome sequencing data. Briefings in Bioinformatics 15:256-278.

Pirooznia M, Kramer M, Parla J, Goes F, Potash J, McCombie W, and Zandi P. 2014. Validation and assessment of variant calling pipelines for next-generation sequencing. Human Genomics 8:14.

Roberts ND, Kortschak RD, Parker WT, Schreiber AW, Branford S, Scott HS, Glonek G, and Adelson DL. 2013. A comparative analysis of algorithms for somatic SNV detection in cancer. Bioinformatics 29:2223-2230.

Schmitt MW, Kennedy SR, Salk JJ, Fox EJ, Hiatt JB, and Loeb LA. 2012. Detection of ultra-rare mutations by next-generation sequencing. Proceedings of the National Academy of Sciences 109:14508-14513.

Sherry ST, Ward M-H, Kholodov M, Baker J, Phan L, Smigielski EM, and Sirotkin K. 2001. dbSNP: the NCBI database of genetic variation. Nucleic Acids Research 29:308-311.

Talwalkar A, Liptrap J, Newcomb J, Hartl C, Terhorst J, Curtis K, Bresler Ma, Song YS, Jordan MI, and Patterson D. 2014. SMaSH: a benchmarking toolkit for human genome variant calling. Bioinformatics.

The 1000 Genomes Project Consortium. 2012. An integrated map of genetic variation from 1,092 human genomes. Nature 491:56-65.

Van der Auwera GA, Carneiro MO, Hartl C, Poplin R, del Angel G, Levy-Moonshine A, Jordan T, Shakir K, Roazen D, Thibault J, Banks E, Garimella KV, Altshuler D, Gabriel S, and DePristo MA. 2002. From FastQ Data to High-Confidence Variant Calls: The Genome Analysis Toolkit Best Practices Pipeline. Current Protocols in Bioinformatics: John Wiley \& Sons, Inc.

Wang K, Li M, and Hakonarson H. 2010. ANNOVAR: functional annotation of genetic variants from high-throughput sequencing data. Nucleic Acids Research 38:e164.

Wang Q, Jia P, Li F, Chen H, Ji H, Hucks D, Dahlman K, Pao W, and Zhao Z. 2013. Detecting somatic point mutations in cancer genome sequencing data: a comparison of mutation callers. Genome Medicine 5:91.

Worthey EA. 2013. Analysis and Annotation of Whole-Genome or Whole-Exome Sequencing-Derived Variants for Clinical Diagnosis. Current Protocols in Human Genetics: John Wiley \& Sons, Inc.

Xu H, DiCarlo J, Satya R, Peng Q, and Wang Y. 2014. Comparison of somatic mutation calling methods in amplicon and whole exome sequence data. BMC Genomics $15: 244$.

Yi M, Zhao Y, Jia L, He M, Kebebew E, and Stephens RM. 2014. Performance comparison of SNP detection tools with illumina exome sequencing data-an assessment using both family pedigree information and sample-matched SNP array data. Nucleic Acids Research 42:e101.

Yu X, and Sun S. 2013. Comparing a few SNP calling algorithms using low-coverage sequencing data. BMC Bioinformatics 14:274.

Zook JM, Chapman B, Wang J, Mittelman D, Hofmann O, Hide W, and Salit M. 2014. Integrating human sequence data sets provides a resource of benchmark SNP and indel genotype calls. Nat Biotech 32:246-251. 
PeerJ reviewing PDF | (v2014:06:2253:2:1:NEW 9 Sep 2014) 


\section{Figure 1}

Recovery of 1KG 454 PCR Validated SNPs (chr20)

A pooled set of 35 validated variant from the 1000 Genomes exome sample characterized in this study ( $n=12)$ was used to assess the sensitivity of various variant calling algorithms. The 1000 Genomes targeted exon samples were not compared because no validated variants were covered in targeted regions for that design. Seven variant calling strategies were tested (GATK UnifiedGenotyper and HaplotypeCaller, with and without filtering low quality variants; VarScan with 3 sets of parameters, see Methods). "VarScan-Cons" is the most conservative set of parameters for VarScan. Each variant caller was also tested with 4 preprocessing conditions: variants called using both GATK indel realignment and quality score recalibration ("Full Pipeline" - purple), indel realignment only ("Realign Only" - red), quality score recalibration only ("Recalibrate Only" - green), or neither ("No Preprocess" - blue). Publically available validated variants are only available for chr20, so this is the maximum number of validated SNPs that can be characterized for these samples. The validation status for each individual SNP under each variant calling condition is provided in Table S7. Validated variants were never called for chr20:3193991 for individual NA18505 (exome sample SRX237141, covered $81 x$ with the reference allele in all reads) or for chr20:57769739 for individual NA18532 (exome sample ERR031956, not covered by any reads but located in the coding sequence for ZNF831). 


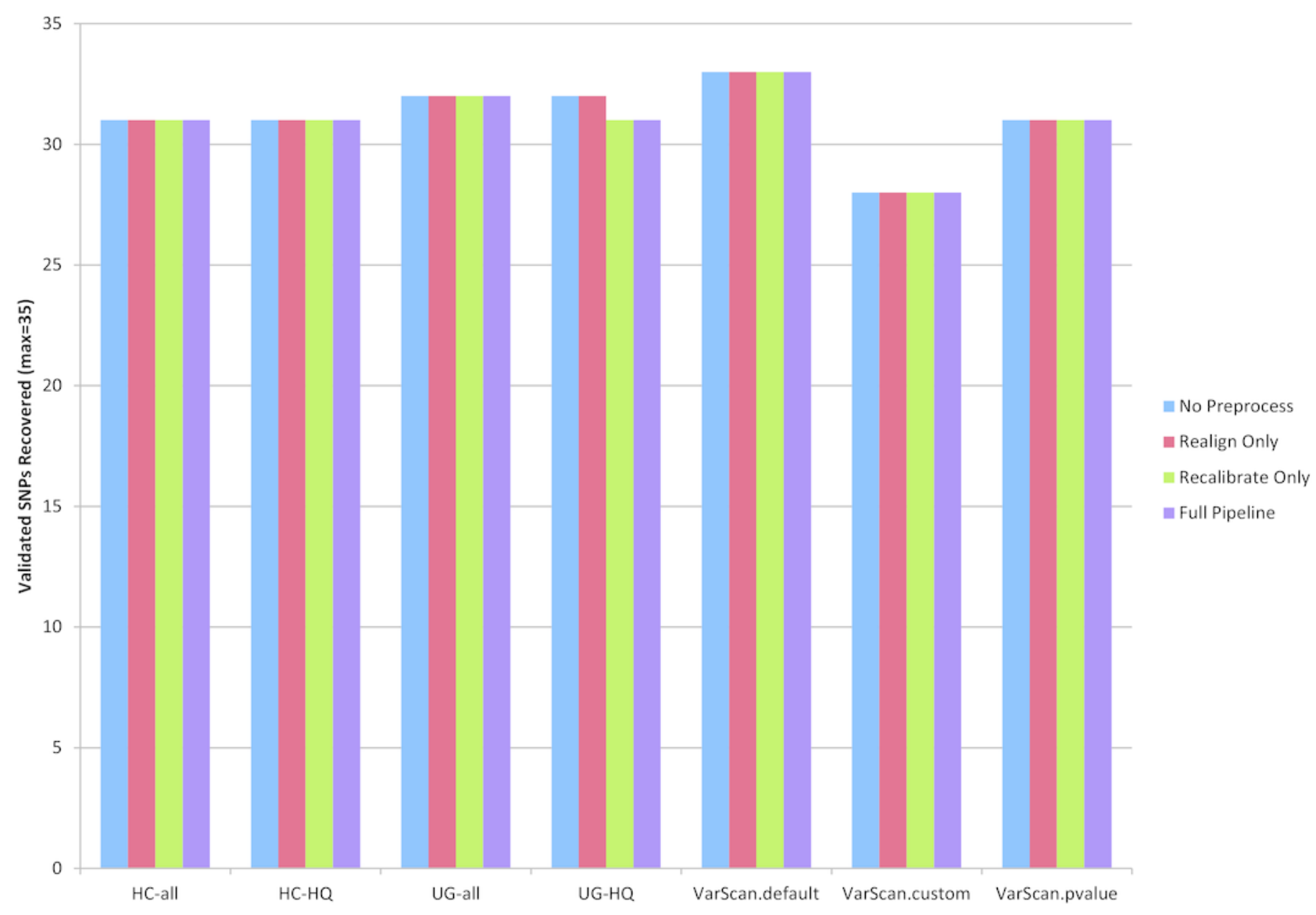




\section{Figure 2}

Targeted Exon Technical Replicate Indel Concordance (1KG - chr20)

Two subjects (NA18510 and NA18637) had two different targeted exon samples.

Concordance between these two samples (per subject) is shown for all SNPs called on chromosome 20. Seven variant calling strategies were tested (GATK UnifiedGenotyper and HaplotypeCaller, with and without filtering low quality variants; VarScan with 3 sets of parameters, see Methods). "VarScan-Cons" is the most conservative set of parameters for VarScan. Each variant caller was also tested with 4 pre-processing conditions: variants called using both GATK indel realignment and quality score recalibration ("Full Pipeline" - purple), indel realignment only ("Realign Only" - red), quality score recalibration only ("Recalibrate Only" - green), or neither ("No Preprocess" - blue). Unlike the SNP calls, coding and targeted variant concordance is not provided for indels because there are essentially no indels called within coding regions on chromosome 20 .

A.

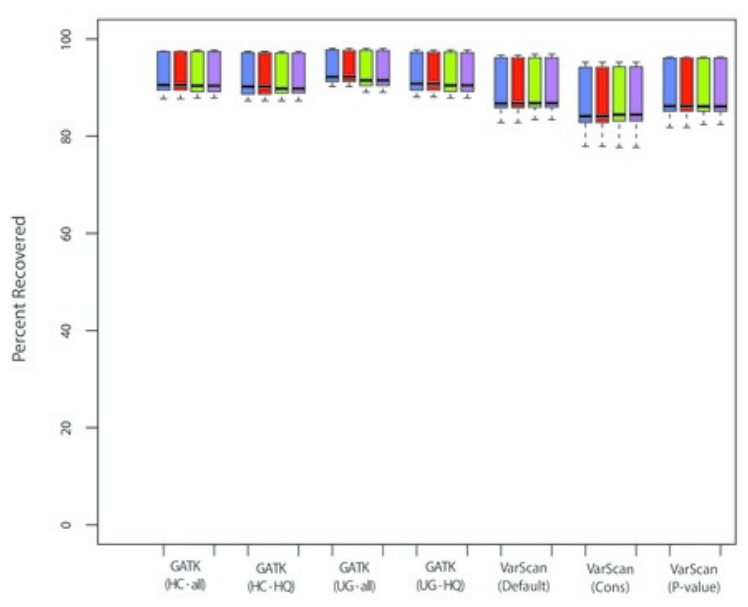

B.

SRP019719 Human610 ( $n=1)$

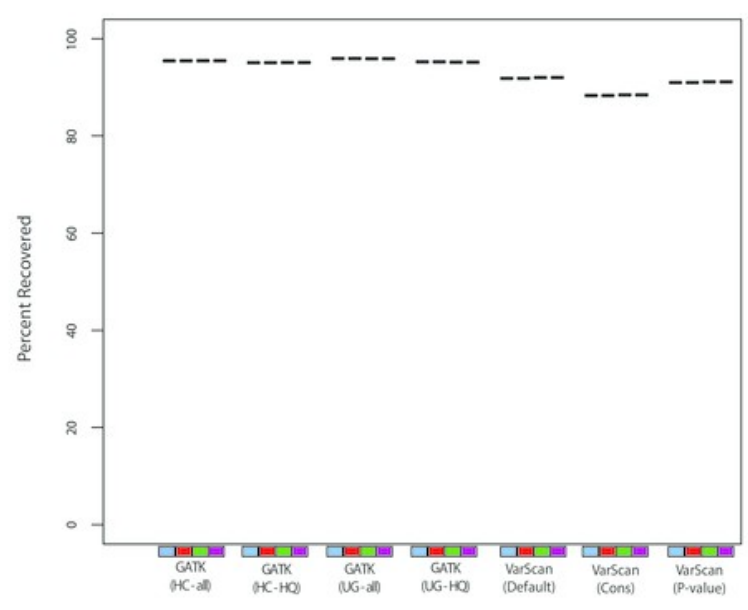

= No Preprocess w Realign Only thecaliturate onst a Full Pipeline 


\title{
Figure 3
}

Recovery of Coding SNPs from Targeted Exon Samples (1KG Exome - chr20)

\begin{abstract}
A.SNP calls from paired targeted exon and exome datasets were compared to test the
\end{abstract} robustness of the calls made in the targeted exon data. Indel calls are not presented because there are almost no coding indels on chr20 for the targeted exon datasets. Two subjects have two targeted exon datasets (Table S1-S2), and concordance with exome datasets was reported for both targeted exon datasets separately (resulting in 14 concordance values per variant calling strategy). Seven variant calling strategies were tested (GATK UnifiedGenotyper and HaplotypeCaller, with and without filtering low quality variants; VarScan with 3 sets of parameters, see Methods). "VarScan-Cons" is the most conservative set of parameters for VarScan. Each variant caller was also tested with 4 preprocessing conditions: variants called using both GATK indel realignment and quality score recalibration ("Full Pipeline" - purple), indel realignment only ("Realign Only" - red), quality score recalibration only ("Recalibrate Only" - green), or neither ("No Preprocess" - blue). Concordance is reported as recovery of SNPs called in the targeted exon data, but these cannot be treated as "gold standard" variant calls. Most clearly, there was a high false positive rate when running VarScan with default parameters, so a high proportion of those variants called in the targeted exon samples could not be recovered in the exome dataset. In fact, on-target coverage is typically lower for the targeted exon samples than the exome staples (Table S1-S2).B.Same as A, but only previously observed variants are included in the concordance calculations. 
A.

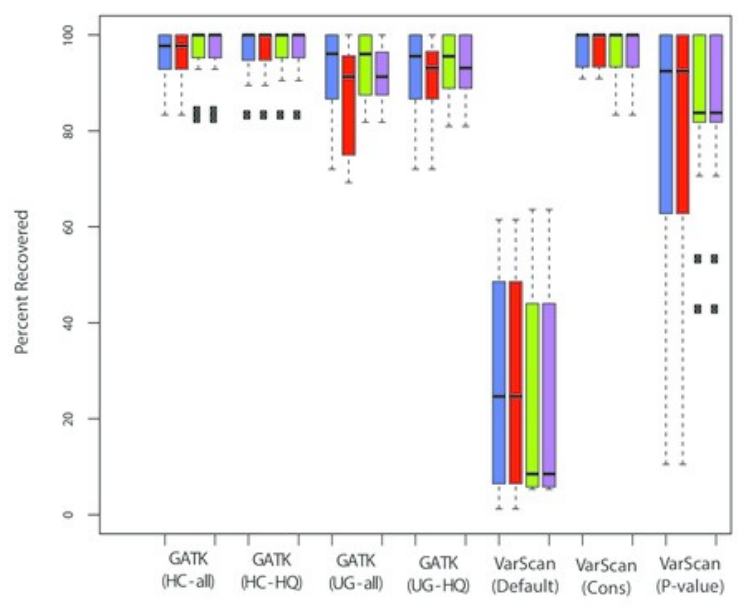

B.

Previously Observed Variants

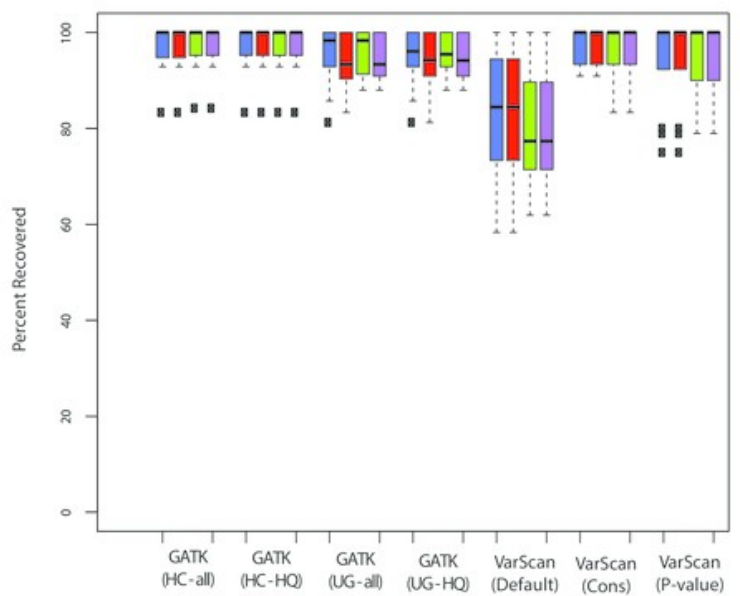




\section{Figure 4}

Frequency of Reads Supporting Coding VarScan-Default SNP Variants (1KG Exome chr20)

Density plots for novel variants (those variants not reported in the 1000 Genomes Project, the Exome Sequencing Project, or dbSNP) are shown with red lines while density plots for previously observed variants are shown with red lines. Notice that most novel variants have a very low frequency of supporting reads, with a trend so strong that it cannot be observed on the scale of the current figure (although a rescaled figure is shown in Figure S4, to emphasize the radically different density distributions). We believe that this because the majority of variants with less than $30 \%$ supporting reads are due to sequencing errors, which is why the height of the peak (in Figure S4) approximately matches the error rate ( 1\%). This distribution looks similar regardless of pre-processing pipeline or target design (e.g. exome versus targeted exon).
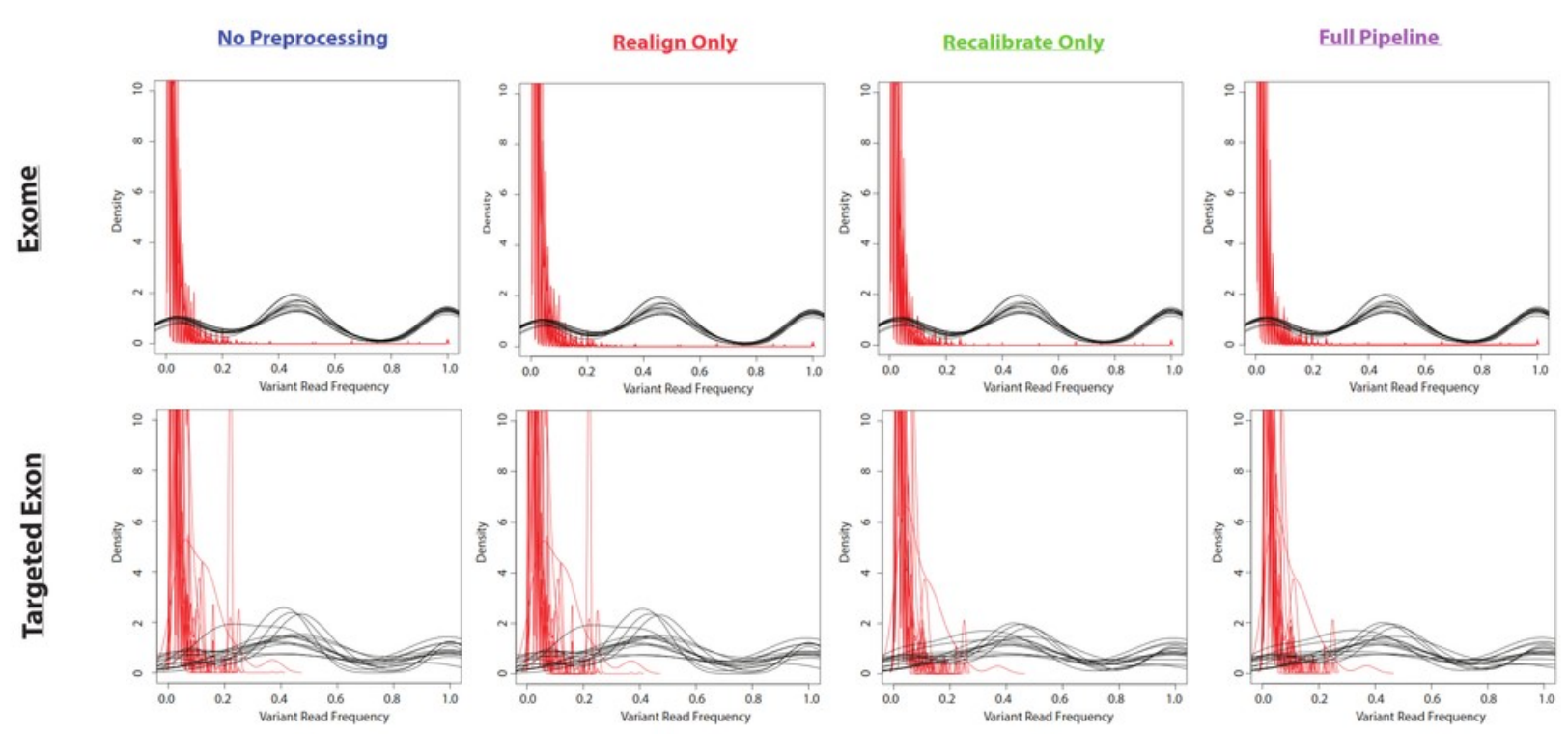

Novel Variants (Absent from 1KG, ESP, dbSNP)

Previously Observed Variants 


\section{Figure 5}

Frequency of Reads Supporting SNP Variants in Exome Datasets

Density plots for novel variants (those variants not reported in the 1000 Genomes Project, the Exome Sequencing Project, or dbSNP) are shown with red lines while density plots for previously observed variants are shown with red lines. Density plots are shown for two datasets (1000 Genomes and SRP019719) with 3 lists of variants (VarScan-Cons, highquality GATK UnifiedGenotyper, and high-quality GATK HaplotypeCaller). Density plots are only created for variant lists with greater than 2 variants, so some samples do not have novel variant density plots. Notice that the high-quality GATK variants lists lack variants with low percentages of variant reads (such as VarScan-Default, Figure 4), similar to VarScan-Cons. This shift in supporting read frequencies correlates with the concordance rates (Figure 3) and the novel variant frequencies (Figure 7) for the corresponding variant lists.
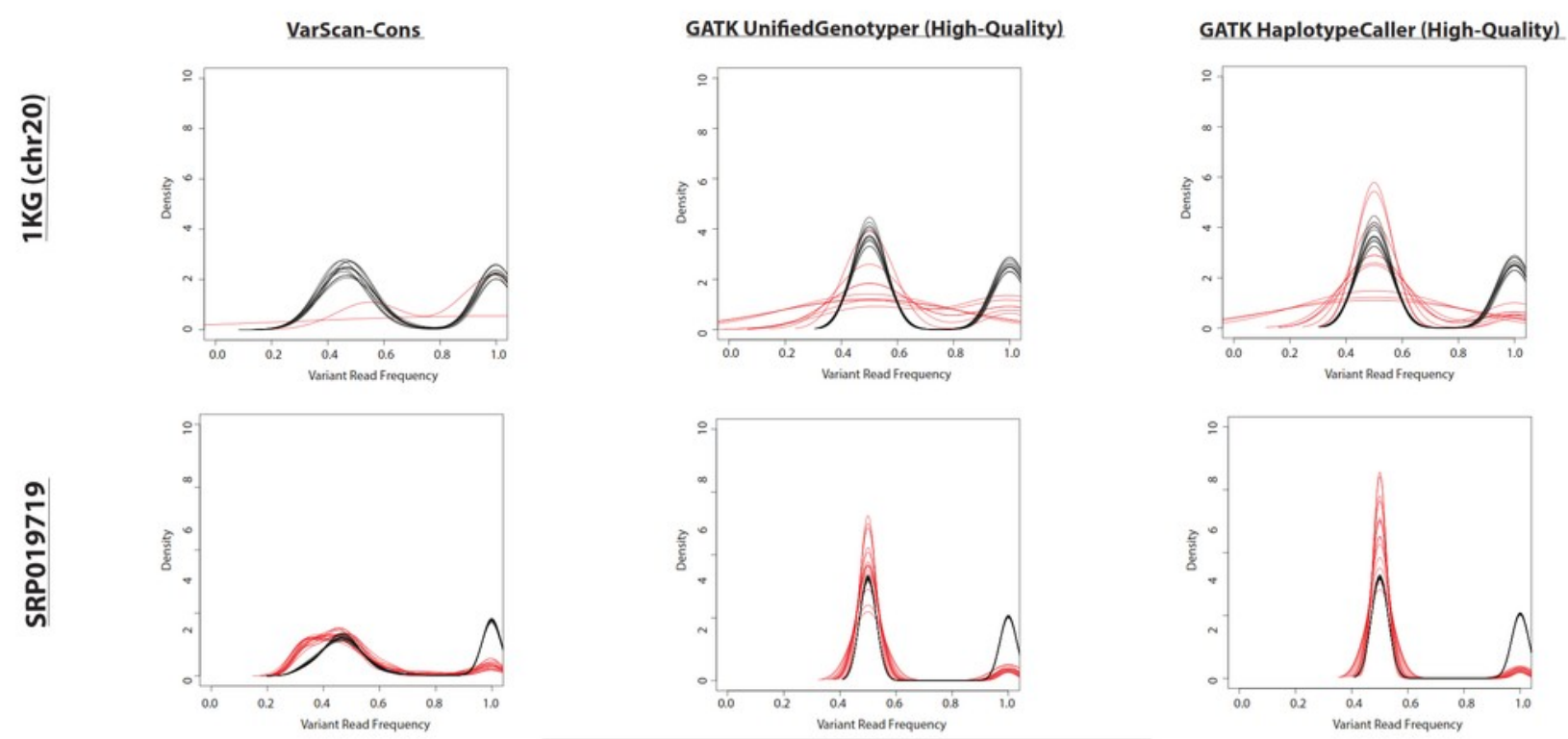

Novel Variants (Absent from 1KG, ESP, dbSNP) 


\section{Figure 6}

Number of Variants Called for GATK versus VarScan (1KG - chr20)

A.Number of SNPs called for selected 1000 Genomes ( $1 K G)$ targeted exon samples $(n=14$, left) and exome samples ( $n=12$, right). The number of SNPs called is displayed for variants called using both GATK indel realignment and quality score recalibration ("Full Pipeline" purple), indel realignment only ("Realign Only" - red), quality score recalibration only ("Recalibrate Only" - green), or neither ("No Preprocess" - blue). Variant counts are provided for the GATK UnifiedGenotyper (“UG”), GATK HaplotypeCaller (“HC”), and VarScan using 3 sets of parameters (see Methods). UnifiedGenotyper and HaplotypeCaller variants are then divided into the set of all variants ("UG-all" and "HC-all") and higher-quality variant calls where variants flagged as low quality have been removed ("UG-HQ" and "HC-HQ"). "VarScan-Cons" is the most conservative set of parameters for VarScan. These values represent the total number of SNPs called.B.Same as A, for indels instead of SNPs. 
A.

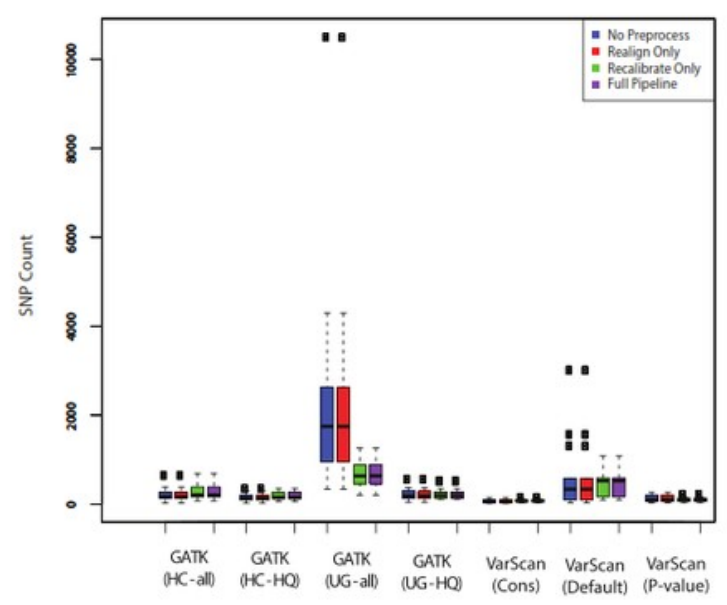

B.

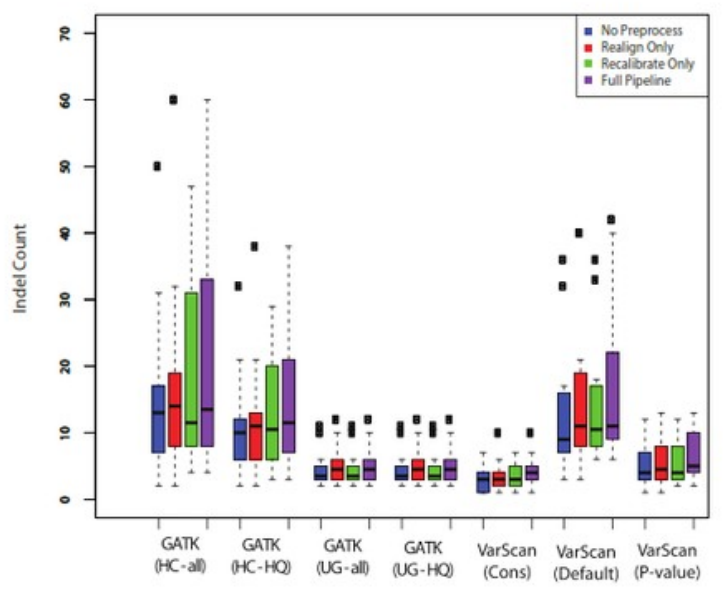

Exome

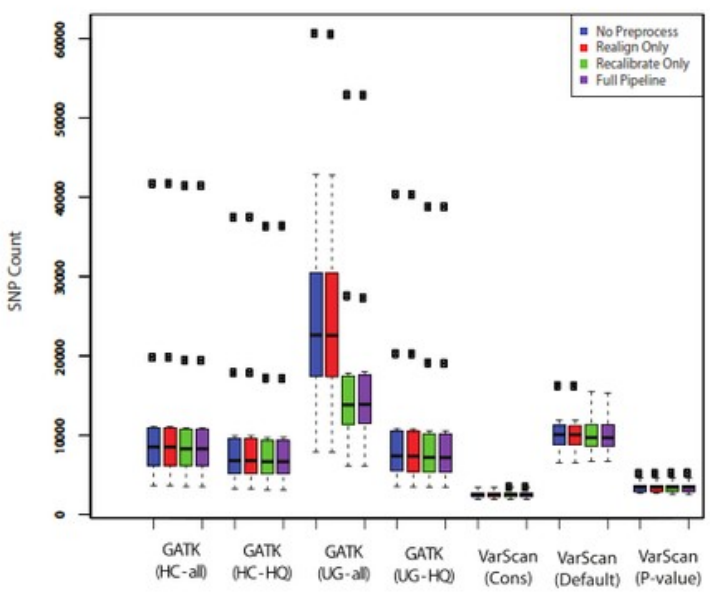

Exome

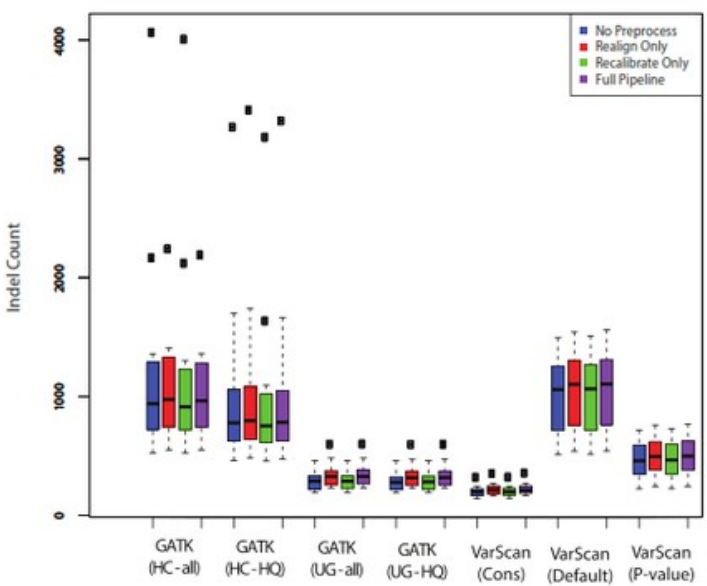




\section{Figure 7}

Distribution of ANNOVAR Annotations for Coding SNP Variants (1KG - chr20)

A. Distribution of variant types defined in the ANNOVAR exome report for selected 1000 Genomes (1KG) targeted exon samples $(n=14)$. Variants are classified based upon population frequency and damaging prediction (see Methods). Low frequency variants (MAF $<0.01)$ that are displayed in orange if they are predicted to be damaging and are displayed in green if they are not predicted to be damaging. Novel variants are displayed in red if they are predicted to be damaging and are displayed in blue if they are not predicted to be damaging. Although all samples should contain some novel variants, a high proportion of novel variants are expected to correlate with a high false positive rate. Seven variant calling strategies were tested (GATK UnifiedGenotyper and HaplotypeCaller, with and without filtering low quality variants; VarScan with 3 sets of parameters, see Methods). "VarScan-Cons" is the most conservative set of parameters for VarScan. Each variant caller was also tested with 4 preprocessing conditions, corresponding to the colored boxes under the bar plot: variants called using both GATK indel realignment and quality score recalibration (purple), indel realignment only (red), quality score recalibration only (green), or neither (blue).B.Same as A, but for selected 1000 Genomes exome samples ( $n=12$, Table S2). 
A.

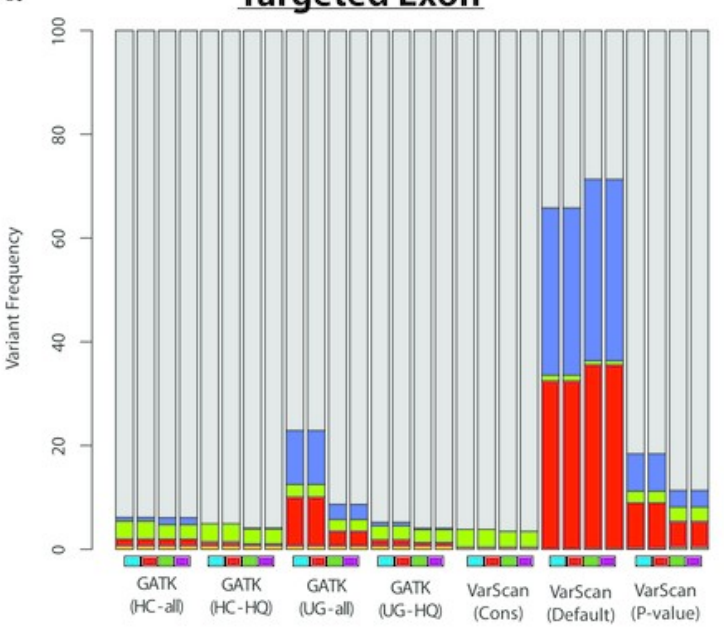

B.

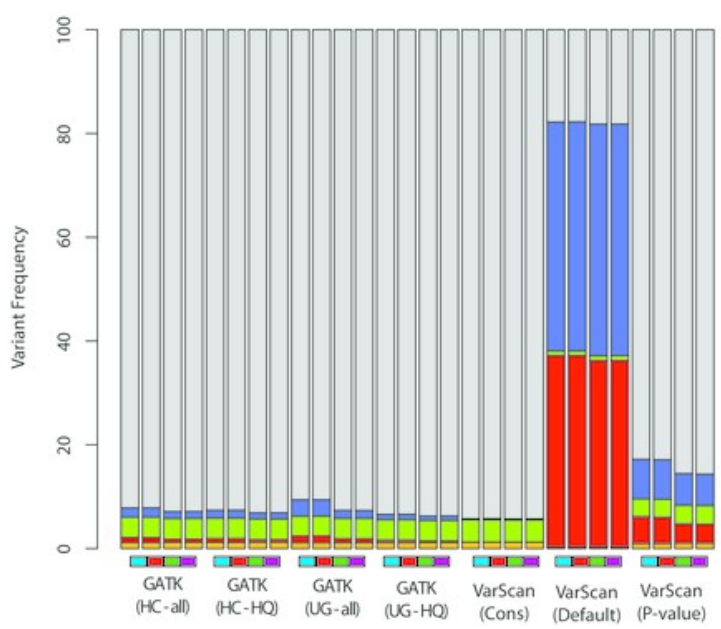

17. Common Variants

- Novel Variants (Not Damaging) Low Frequency Variants (Not Damaging)

- Novel Variants

Low Frequency Variants 


\section{Figure 8}

\section{Exome Variant Caller Overlap (Coding Variants)}

All coding variants were tabulated for all exome samples ( $1 K G n=12$, left; SRP019719 $n=15$, right). Only coding variants on chromosome 20 were considered for the 1000 Genomes (1KG) samples, but all coding variants were considered for the SRP019719 samples. In order to simplify presentation of these results, we focused on the highest quality variant calls for each variant calling strategy: GATK UnifiedGenotyper with low-quality variants removed (UG$H Q$, blue), GATK HaplotypeCaller with low-quality variants removed (HC-HQ, green), and VarScan using a custom set of conservative parameters (VarScan-Cons, red). Similarly, only variants subject to GATK indel realignment and quality score recalibration ("Full Pipeline") are considered for this comparison. To show the different concordance rates, SNPs are presented at the figure and indels are presented at the bottom of the figure. Almost all VarScan-Cons variants were also called by GATK (either HaplotypeCaller or UnifiedGenotyper). All three variant callers called a similar number of SNPs, but GATK HaplotyperCaller called more indels than either GATK UnifiedGenotyper or VarScan-Cons. 


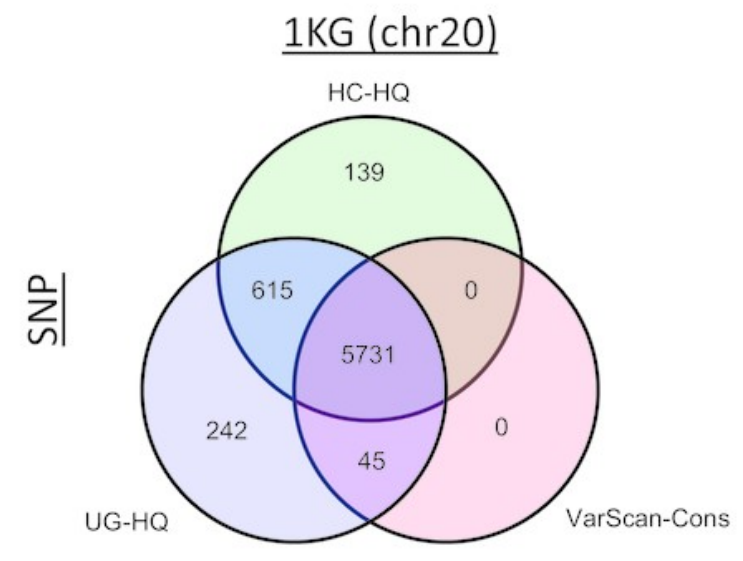

$\underline{\text { SRP019719 (all) }}$
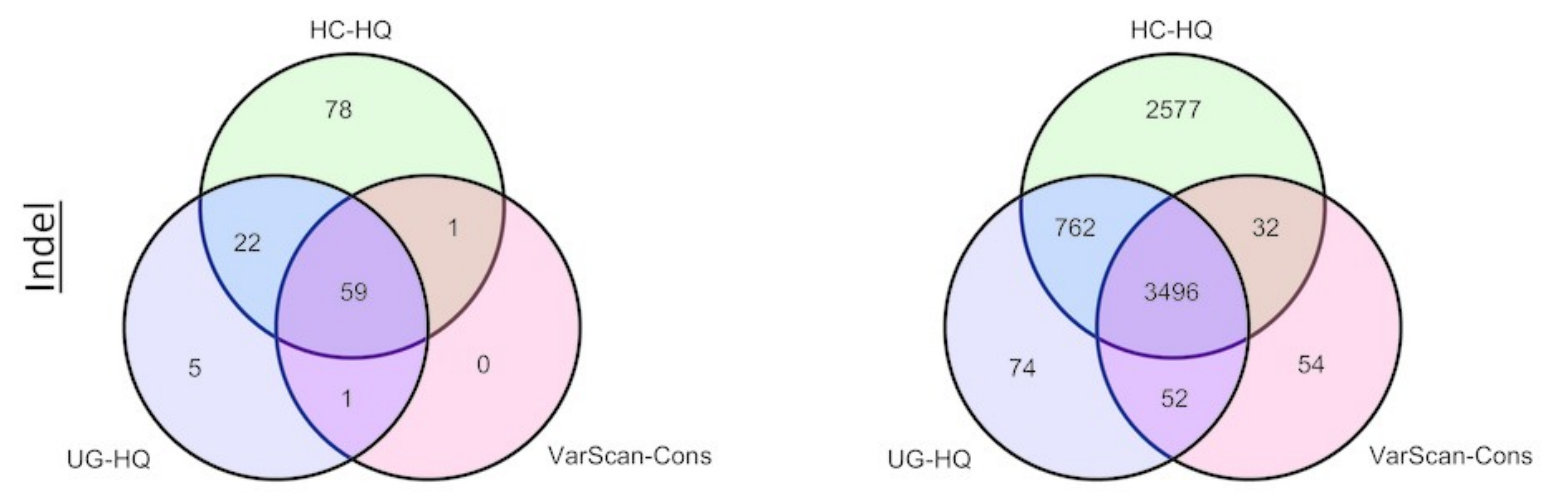


\section{Figure 9}

QC Metrics to Estimate Specificity Versus Sensitivity for Variant Callers (Coding SNPs)

For each of the three datasets characterized in this study (1KG targeted exon, $n=14 ; 1 \mathrm{KG}$ exome, $n=12$; SRP019719 exome, $n=15$ ), the number of coding SNPs called per sample is plotted along the $x$-axis and the proportion of novel variants is plotted on the $y$-axis. In order to simplify presentation of these results, we focused on the highest quality variant calls for each variant calling strategy: GATK UnifiedGenotyper with low-quality variants removed (UG$H Q$, blue), GATK HaplotypeCaller with low-quality variants removed (HC-HQ, green), and VarScan using a custom set of conservative parameters (VarScan-Cons, red). Additionally, an unfiltered set of variants called via samtools are plotted in black. Only variants subject to GATK indel realignment and quality score recalibration ("Full Pipeline") are considered for this comparison. The shape of the data point corresponds to the depth of on-target coverage: $<50 x$ coverage is represented as an $X$ in an open-circle, 50-100x is represented as an open circle, and $>100 x$ is represented as a filled circle. If the novel percentage was tightly correlated with the actual false positive rate and the number of variants was tightly correlated with the actual sensitivity of the variant caller, than the ideal variant caller would show a cluster of data points in the bottom-right hand corner of the plot.
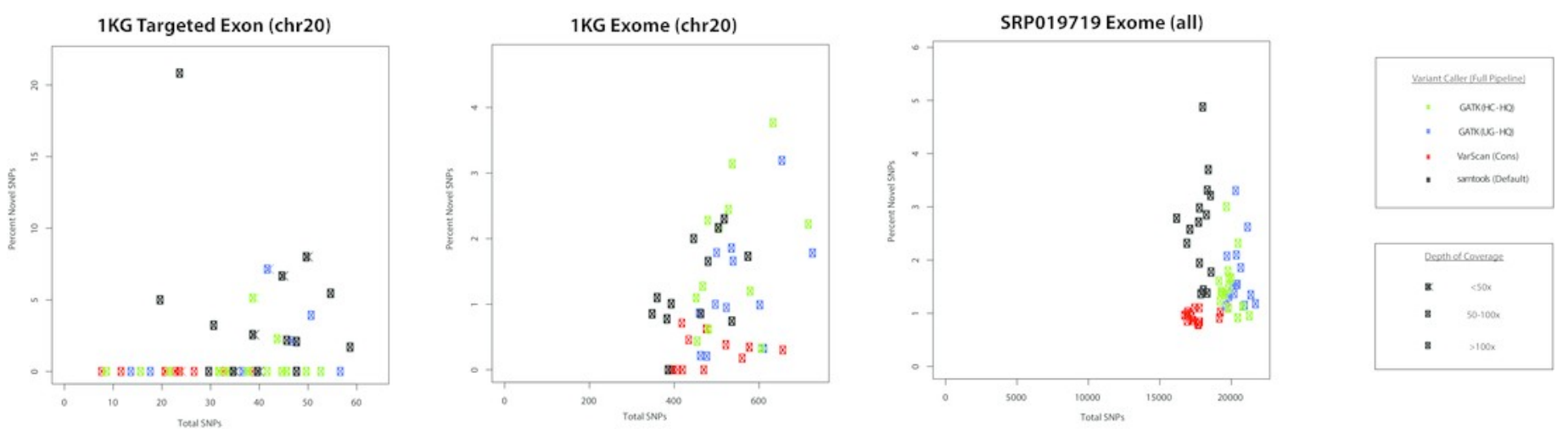


\section{Table 1 (on next page)}

Parameter Settings for VarScan Comparisons

Unless a p-value cutoff is specified, VarScan doesn't calculate p-values. 
Table 1: Parameter Settings for VarScan Comparisons

\begin{tabular}{|l|c|c|c|}
\hline & VarScan-Default & VarScan-Pvalue & VarScan-Cons \\
\hline Minimum Coverage & 8 & 8 & 10 \\
\hline $\begin{array}{l}\text { Minimum Supporting } \\
\text { Reads }\end{array}$ & 2 & 2 & 4 \\
\hline $\begin{array}{l}\text { Minimum Average } \\
\text { Quality }\end{array}$ & 15 & 15 & 20 \\
\hline $\begin{array}{l}\text { Minimum Variant } \\
\text { Frequency }\end{array}$ & 0.01 & 0.01 & 0.3 \\
\hline Maximum P-Value & [none] & 0.05 & [none] \\
\hline
\end{tabular}

Unless a p-value cutoff is specified, VarScan doesn't calculate p-values. 